\title{
MORTAR SPECTRAL ELEMENT DISCRETIZATION OF THE LAPLACE AND DARCY EQUATIONS WITH DISCONTINUOUS COEFFICIENTS
}

\author{
Zakaria Belhachmi $^{1}$, Christine Bernardi ${ }^{2}$ And Andreas Karageorghis ${ }^{3}$
}

\begin{abstract}
This paper deals with the mortar spectral element discretization of two equivalent problems, the Laplace equation and the Darcy system, in a domain which corresponds to a nonhomogeneous anisotropic medium. The numerical analysis of the discretization leads to optimal error estimates and the numerical experiments that we present enable us to verify its efficiency.
\end{abstract}

Mathematics Subject Classification. 65N35, 65N55.

Received June 9, 2006. Revised April 13, 2007.

\section{INTRODUCTION}

As a model for the geometry of a nonhomogeneous anisotropic medium, we consider the square $\Omega=]-1,1[2$, divided into three subdomains $\Omega_{1}, \Omega_{2}$ and $\Omega_{3}$ defined by

$$
\left.\Omega_{1}=\right]-1,1[\times] 1-\varepsilon, 1\left[, \quad \Omega_{2}=\right]-1,0[\times]-1,1-\varepsilon\left[, \quad \Omega_{3}=\right] 0,1[\times]-1,1-\varepsilon[
$$

where $\varepsilon$ is a fixed parameter, $0<\varepsilon<1$, as illustrated in Figure 1. We are particularly interested in the anisotropic case where $\varepsilon$ is very small.

We introduce a function $\alpha$ which is equal to a positive constant $\alpha_{k}$ in each subdomain $\Omega_{k}, 1 \leq k \leq 3$, and we consider the following two problems

$$
\begin{gathered}
\begin{cases}-\operatorname{div}(\alpha \operatorname{grad} p)=f-\operatorname{div} \boldsymbol{g} & \text { in } \Omega, \\
\partial_{n} p=0 & \text { on } \partial \Omega,\end{cases} \\
\begin{cases}\mathbf{u}+\alpha \operatorname{grad} p=\boldsymbol{g} & \text { in } \Omega, \\
\operatorname{div} \mathbf{u}=f & \text { in } \Omega, \\
\mathbf{u} \cdot \boldsymbol{n}=0 & \text { on } \partial \Omega .\end{cases}
\end{gathered}
$$

\footnotetext{
Keywords and phrases. Mortar method, spectral elements, Laplace equation, Darcy equation.

${ }^{1}$ L.M.A.M. (UMR 7122), Université Paul Verlaine-Metz, Ile de Saulcy, 57045 Metz Cedex 01, France.

${ }^{2}$ Laboratoire Jacques-Louis Lions, C.N.R.S. \& Université Pierre et Marie Curie, B.C. 187, 4 place Jussieu, 75252 Paris Cedex 05, France. bernardi@ann.jussieu.fr

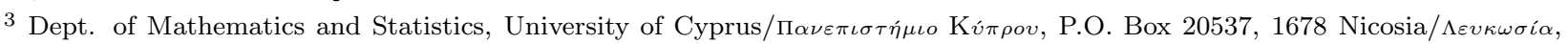
Cyprus/Kúroos.

(c) EDP Sciences, SMAI 2007
} 


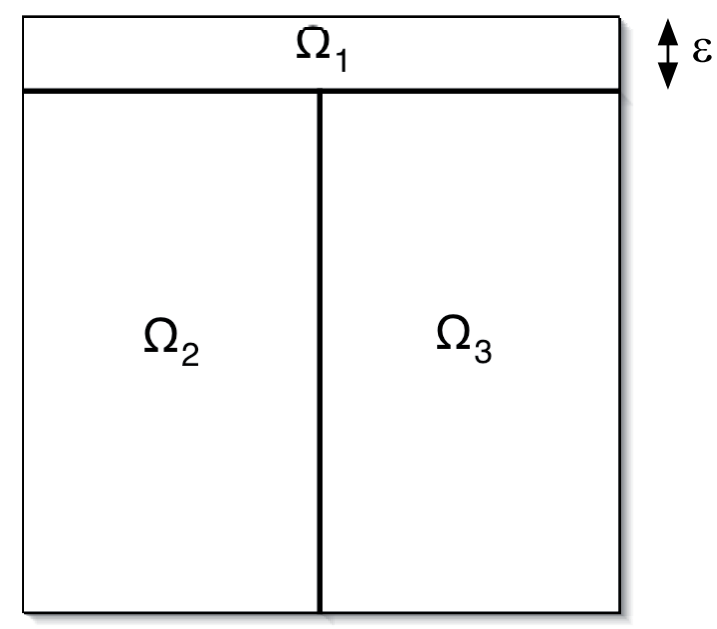

FIGURE 1 . The partition of the domain $\Omega$.

Here $\boldsymbol{n}$ denotes the unit outward normal vector to $\Omega$ on its boundary $\partial \Omega$. From a physical point of view, problem (1.2) is a generalized Laplace equation which, in the case $\boldsymbol{g}=\mathbf{0}$, models the stationary diffusion of the temperature in a nonhomogeneous medium with diffusion coefficient equal to $\alpha_{k}$ on each $\Omega_{k}$ when heated by an internal source $f$. Problem (1.3) is known as the Darcy system and, in the case $f=0$, models the flow of a viscous incompressible fluid in a nonhomogeneous porous medium where $\alpha$ represents the drag coefficient (the different values of $\alpha$ are due to different permeabilities in the subdomains $\Omega_{k}$ ). In both cases, it is interesting to consider the case where the ratio of the maximum of $\alpha$ to its minimum is large and also the case where $\varepsilon$ is small, which corresponds, for instance, to soil layers for problem (1.3). We refer, among others, to [1], [4] and [15] for the treatment of the piecewise constant coefficient $\alpha$ and to [6] for the treatment of anisotropic subdomains in the spectral element context.

From a mathematical point of view, it is well known that problems (1.2) and (1.3) are equivalent when the data $\boldsymbol{g}$ have a null normal trace on $\partial \Omega$, in the following sense: Problem (1.3) represents a mixed formulation of problem (1.2) where the new unknown $\boldsymbol{u}=\boldsymbol{g}-\alpha \operatorname{grad} p$ is introduced and problem (1.2) is derived from problem (1.3) by simply taking the divergence of the first equation. The main goal of this study is to write the variational formulation of each problem, to propose a discretization of each problem based on this variational formulation via a Galerkin method and to compare them.

The mortar element method, as introduced in [9], is a domain decomposition technique that enables one to work with nonconforming decompositions of the computational domain without overlap. It therefore seems ideally suited for handling the discontinuities of the function $\alpha$. We consider this method in the framework of spectral discretizations and, in order to take into account the anisotropy of the domain $\Omega_{1}$ in the case of very small values of $\varepsilon$, we use different degrees of polynomials in $\Omega_{1}$ in the horizontal and vertical directions. This, combined with the use of quadrature formulæ as is usually done in spectral methods, leads to a discrete problem. We prove that it is well-posed and derive optimal error estimates.

As first proposed in [3], the key idea for the implementation of the mortar element method consists in handling the matching conditions on the interfaces between different subdomains via the introduction of a Lagrange multiplier. We describe the linear system that results from this algorithm and present some numerical experiments that allow us to compare the two formulations. In both cases, the numerical results are in good agreement with the analysis. The error curves are the same for both problems, but the implementation of the discrete problem associated with system (1.2) appears to be less expensive, at least in the basic situations that we consider. 
The outline of the paper is as follows.

- In Section 2, we recall the variational formulations of both problems (1.2) and (1.3) and prove their well-posedness.

- In Section 3, we describe the discrete problems and verify that they have a unique solution.

- Section 4 is devoted to the numerical analysis of these problems. We derive error estimates where the dependence in $\varepsilon$ and the $\alpha_{k}$ is explicitly taken into account.

- In Section 5, we present the algorithm for implementing the mortar element method, together with some numerical experiments in order to compare the two proposed discretizations.

- Some conclusions and possible extensions are proposed in Section 6.

\section{VARIATIONAL FORMULATIONS OF THE PROBLEMS}

The generic point in $\Omega$ is denoted by $\boldsymbol{x}=(x, y)$. Throughout this paper, we use the standard Hilbertian Sobolev spaces $H^{s}(\Omega)$ for all nonnegative values of $s$, equipped with the usual norm $\|\cdot\|_{H^{s}(\Omega)}$, and also their analogues on each $\Omega_{k}$. The space $H^{0}(\Omega)$ is denoted by $L^{2}(\Omega)$. Since the unknown $p$ is clearly defined up to an additive constant in both problems (1.2) and (1.3), we also introduce the space

$$
L_{0}^{2}(\Omega)=\left\{q \in L^{2}(\Omega) ; \int_{\Omega} q(\boldsymbol{x}) \mathrm{d} \boldsymbol{x}=0\right\}
$$

We set:

$$
\alpha_{\min }=\min _{1 \leq k \leq 3} \alpha_{k}, \quad \alpha_{\max }=\max _{1 \leq k \leq 3} \alpha_{k},
$$

and we recall that $\alpha_{\min }$ is positive. We also assume without restriction that the data $(f, \boldsymbol{g})$ satisfy

$$
f \in L_{0}^{2}(\Omega), \quad \boldsymbol{g} \in L^{2}(\Omega)^{2}, \quad \operatorname{div} \boldsymbol{g} \in L^{2}(\Omega), \quad \boldsymbol{g} \cdot \boldsymbol{n}=0 \quad \text { on } \partial \Omega .
$$

The variational formulation of the Laplace equation is straightforward:

Find $p$ in $\left.H^{1}(\Omega) \cap L_{0}^{2}(\Omega)\right)$ such that

$$
\forall q \in H^{1}(\Omega), \quad a_{L}(p, q)=\int_{\Omega}(f q+\boldsymbol{g} \cdot \operatorname{grad} q)(\boldsymbol{x}) \mathrm{d} \boldsymbol{x},
$$

where the bilinear form $a_{L}(\cdot, \cdot)$ is defined by

$$
a_{L}(p, q)=\sum_{k=1}^{3} \alpha_{k} \int_{\Omega_{k}} \operatorname{grad} p \cdot \operatorname{grad} q \mathrm{~d} \boldsymbol{x} .
$$

It is readily checked that, when assumption (2.3) holds, equation (2.4) can equivalently be enforced for all $q$ in $H^{1}(\Omega)$ or for all $q$ in $H^{1}(\Omega) \cap L_{0}^{2}(\Omega)$. Moreover, it follows from the density of the space $\mathscr{C}^{\infty}(\bar{\Omega})$ of indefinitely differentiable functions on $\bar{\Omega}$ in $H^{1}(\Omega)$ that the variational formulation (2.4) is equivalent to problem (1.2).

A consequence of the Bramble-Hilbert inequality is the ellipticity property

$$
\forall q \in H^{1}(\Omega) \cap L_{0}^{2}(\Omega), \quad a_{L}(q, q) \geq c \alpha_{\min }\|q\|_{H^{1}(\Omega)}^{2}
$$

Thus the well-posedness of problem (2.4) follows by applying the Lax-Milgram lemma.

Proposition 2.1. For any data $(f, \boldsymbol{g})$ satisfying $(2.3)$, problem $(2.4)$ has a unique solution $p$ in $H^{1}(\Omega) \cap L_{0}^{2}(\Omega)$. 
In the sequel, since we want to optimize our estimates with respect to the ratio $\alpha_{\max } / \alpha_{\min }$, we work with the energy norm

$$
\|q\|_{1, \alpha}=\left(\sum_{k=1}^{3} \alpha_{k}\|\operatorname{grad} q\|_{L^{2}\left(\Omega_{k}\right)^{2}}^{2}\right)^{\frac{1}{2}} .
$$

The solution $p$ of problem (2.4) thus satisfies the following stability property

$$
\|p\|_{1, \alpha} \leq \alpha_{\min }^{-\frac{1}{2}}\left(c\|f\|_{L^{2}(\Omega)}+\|\boldsymbol{g}\|_{L^{2}(\Omega)^{2}}\right) .
$$

Concerning system (1.3), we recall from [2], Section 2.1, and [10], Section XIII.1, for instance, that it admits two variational formulations: The boundary conditions in the third line of (1.3) can be treated as essential or natural ones or, equivalently, the unknown $p$ can be sought for in $L^{2}(\Omega)$ or in $H^{1}(\Omega)$. We choose to work with the latter formulation which allows for easier comparisons with problem (2.4) since $p$ belongs to the same space. In order to work with a symmetric formulation, we also observe that the first line in (1.3) can be written equivalently as

$$
\alpha_{k}^{-1} \boldsymbol{u}+\operatorname{grad} p=\alpha_{k}^{-1} \boldsymbol{g} \quad \text { in } \Omega_{k}, 1 \leq k \leq 3 .
$$

The resulting variational formulation is

Find $(\boldsymbol{u}, p)$ in $L^{2}(\Omega)^{2} \times\left(H^{1}(\Omega) \cap L_{0}^{2}(\Omega)\right)$ such that

$$
\begin{aligned}
\forall \boldsymbol{v} \in L^{2}(\Omega)^{2}, \quad a_{D}(\boldsymbol{u}, \boldsymbol{v})+b_{D}(\boldsymbol{v}, p) & =\sum_{k=1}^{3} \alpha_{k}^{-1} \int_{\Omega_{k}}(\boldsymbol{g} \cdot \boldsymbol{v})(\boldsymbol{x}) \mathrm{d} \boldsymbol{x} \\
\forall q \in H^{1}(\Omega), \quad b_{D}(\boldsymbol{u}, q) & =-\int_{\Omega}(f q)(\boldsymbol{x}) \mathrm{d} \boldsymbol{x}
\end{aligned}
$$

where the bilinear forms $a_{D}(\cdot, \cdot)$ and $b_{D}(\cdot, \cdot)$ are defined by

$$
a_{D}(\boldsymbol{u}, \boldsymbol{v})=\sum_{k=1}^{3} \alpha_{k}^{-1} \int_{\Omega_{k}}(\boldsymbol{u} \cdot \boldsymbol{v})(\boldsymbol{x}) \mathrm{d} \boldsymbol{x}, \quad b_{D}(\boldsymbol{v}, q)=\int_{\Omega} \boldsymbol{v} \cdot \operatorname{grad} q \mathrm{~d} \boldsymbol{x} .
$$

Here also, the equivalence of the variational formulation (2.10) with system (1.3) (see also (2.9)) follows from the density of $\mathscr{C}^{\infty}(\bar{\Omega})$ in $H^{1}(\Omega)$ and also in $L^{2}(\Omega)$.

Proving the well-posedness of problem (2.10) relies on the standard arguments for saddle-point problems. The space $L^{2}(\Omega)^{2}$ is now equipped with the norm

$$
\|\boldsymbol{v}\|_{0, \alpha^{-1}}=\left(\sum_{k=1}^{3} \alpha_{k}^{-1}\|\boldsymbol{v}\|_{L^{2}\left(\Omega_{k}\right)}^{2}\right)^{\frac{1}{2}}
$$

and the space $H^{1}(\Omega)$ with the norm defined in (2.7). First, we have the ellipticity property

$$
\forall \boldsymbol{v} \in L^{2}(\Omega)^{2}, \quad a_{D}(\boldsymbol{v}, \boldsymbol{v})=\|\boldsymbol{v}\|_{0, \alpha^{-1}}^{2} .
$$

Further, by taking $\boldsymbol{v}$ equal to $\alpha \operatorname{grad} q$, we obtain

$$
b_{D}(\boldsymbol{v}, q)=\|q\|_{1, \alpha}^{2} \quad \text { and } \quad\|\boldsymbol{v}\|_{0, \alpha^{-1}}=\|q\|_{1, \alpha},
$$


whence the inf-sup condition

$$
\forall q \in H^{1}(\Omega) \cap L_{0}^{2}(\Omega), \quad \sup _{\boldsymbol{v} \in L^{2}(\Omega)^{2}} \frac{b_{D}(\boldsymbol{v}, q)}{\|\boldsymbol{v}\|_{0, \alpha^{-1}}} \geq\|q\|_{1, \alpha} .
$$

Finally, we introduce the kernel

$$
V=\left\{\boldsymbol{v} \in L^{2}(\Omega)^{2} ; \forall q \in H^{1}(\Omega), b_{D}(\boldsymbol{v}, q)=0\right\} .
$$

It can be readily verified that $V$ is the space of functions $\boldsymbol{v}$ in $L^{2}(\Omega)^{2}$ such that

$$
\operatorname{div} \boldsymbol{v}=0 \quad \text { in } \Omega \quad \text { and } \quad \boldsymbol{v} \cdot \boldsymbol{n}=0 \quad \text { on } \partial \Omega .
$$

Thus we are now in a position to prove the well-posedness of problem (2.10), from the arguments given for instance in [14], Chapter 1, Section 4.1.

Proposition 2.2. For any data $(f, \boldsymbol{g})$ satisfying $(2.3)$, problem $(2.10)$ has a unique solution $(\boldsymbol{u}, p)$ in $L^{2}(\Omega)^{2} \times$ $\left(H^{1}(\Omega) \cap L_{0}^{2}(\Omega)\right)$. Moreover this solution satisfies

$$
\|\boldsymbol{u}\|_{0, \alpha^{-1}}+\|p\|_{1, \alpha} \leq 2 \alpha_{\min }^{-\frac{1}{2}}\left(c\|f\|_{L^{2}(\Omega)}+2\|\boldsymbol{g}\|_{L^{2}(\Omega)^{2}}\right)
$$

Proof. We first verify that the solution is unique. When taking $(f, \boldsymbol{g})$ equal to zero, we observe that $\boldsymbol{u}$ belongs to $V$. Taking $\boldsymbol{v}$ equal to $\boldsymbol{u}$ in the first line of (2.10) and using (2.13) yield that $\boldsymbol{u}$ is zero. Further, $p$ is also zero from (2.14), which proves the uniqueness result. The existence and stability estimates are then derived by applying three times [14], Chapter 1, Lemma 4.1.

1) By combining this lemma with the inf-sup condition (2.14), we derive the existence of a function $\boldsymbol{u}^{*}$ in $L^{2}(\Omega)^{2}$ such that

$$
\forall q \in H^{1}(\Omega) \cap L_{0}^{2}(\Omega), \quad b_{D}\left(\boldsymbol{u}^{*}, q\right)=-\int_{\Omega}(f q)(\boldsymbol{x}) \mathrm{d} \boldsymbol{x} \quad \text { and } \quad\left\|\boldsymbol{u}^{*}\right\|_{0, \alpha^{-1}} \leq c \alpha_{\min }^{-\frac{1}{2}}\|f\|_{L^{2}(\Omega)},
$$

where $c$ is the constant appearing in the Bramble-Hilbert inequality. The first equation in (2.18) obviously holds for all $q$ in $H^{1}(\Omega)$.

2) Because of the choice of $\boldsymbol{u}^{*}$, the function $\boldsymbol{u}^{0}=\boldsymbol{u}-\boldsymbol{u}^{*}$ belongs to $V$ and the first equation in (2.10) can be written as

$$
\forall \boldsymbol{v} \in V, \quad a_{D}\left(\boldsymbol{u}^{0}, \boldsymbol{v}\right)=\sum_{k=1}^{3} \alpha_{k}^{-1} \int_{\Omega_{k}} \boldsymbol{g} \cdot \boldsymbol{v} \mathrm{d} \boldsymbol{x}-a_{D}\left(\boldsymbol{u}^{*}, \boldsymbol{v}\right) .
$$

From (2.13) and the Lax-Milgram lemma, equation (2.19) has a unique solution $\boldsymbol{u}^{0}$, which moreover satisfies

$$
\left\|\boldsymbol{u}^{0}\right\|_{0, \alpha^{-1}} \leq \alpha_{\min }^{-\frac{1}{2}}\|\boldsymbol{g}\|_{L^{2}(\Omega)^{2}}+\left\|\boldsymbol{u}^{*}\right\|_{0, \alpha^{-1}}
$$

3) The pressure $p$ must now satisfy the equation

$$
\forall \boldsymbol{v} \in L^{2}(\Omega)^{2}, \quad b_{D}(\boldsymbol{v}, p)=\sum_{k=1}^{3} \alpha_{k}^{-1} \int_{\Omega_{k}} \boldsymbol{g} \cdot \boldsymbol{v} \mathrm{d} \boldsymbol{x}-a_{D}\left(\boldsymbol{u}^{0}+\boldsymbol{u}^{*}, \boldsymbol{v}\right) .
$$

Since the right-hand side of the previous equation vanishes for all functions $\boldsymbol{v}$ in $V$ because of (2.19), the existence of a $p$ satisfying this equation follows from (2.14), together with the estimate

$$
\|p\|_{1, \alpha} \leq \alpha_{\min }^{-\frac{1}{2}}\|\boldsymbol{g}\|_{L^{2}(\Omega)^{2}}+\left\|\boldsymbol{u}^{0}\right\|_{0, \alpha^{-1}}+\left\|\boldsymbol{u}^{*}\right\|_{0, \alpha^{-1}}
$$


As a consequence, the pair $\left(\boldsymbol{u}=\boldsymbol{u}^{0}+\boldsymbol{u}^{*}, p\right)$ is a solution of problem (2.10) and estimate (2.17) follows from (2.18), $(2.20)$ and $(2.21)$.

Remark 2.3. Estimate (2.17) is exactly of the same type as (2.8) and the constant $c$ which is involved in it is the same as the constant in (2.8). Such an estimate can also be derived for modified formulations, for instance if the first line of (1.3) were not replaced by (2.9) or if it were multiplied by different powers of $\alpha$. However, proving (2.17) in all these cases requires inf-sup conditions that involve different weighted norms on the solution and the test functions and therefore seems to be less natural.

We now state the equivalence of problems (2.4) and (2.10) in a precise form.

Proposition 2.4. Problems (2.4) and (2.10) are equivalent in the following sense:

(i) For any solution $p$ of problem (2.4), there exists a unique function $\boldsymbol{u}$ in $L^{2}(\Omega)^{2}$ such that the pair $(\boldsymbol{u}, p)$ is a solution of problem (2.10).

(ii) For any solution $(\boldsymbol{u}, p)$ of problem (2.10), the function $p$ is a solution of problem (2.4).

Proof. We check successively assertions (i) and (ii).

1) Let $p$ be a solution of problem (2.4). From (2.13) and the Lax-Milgram lemma, the equation

$$
\forall \boldsymbol{v} \in L^{2}(\Omega)^{2}, \quad a_{D}(\boldsymbol{u}, \boldsymbol{v})=\sum_{k=1}^{3} \alpha_{k}^{-1} \int_{\Omega_{k}} \boldsymbol{g} \cdot \boldsymbol{v} \mathrm{d} \boldsymbol{x}-b_{D}(\boldsymbol{v}, p),
$$

has a unique solution $\boldsymbol{u}$ in $L^{2}(\Omega)^{2}$. Moreover, this solution satisfies, for all $q$ in $H^{1}(\Omega)$,

$$
b_{D}(\boldsymbol{u}, q)=a_{D}(\boldsymbol{u}, \alpha \operatorname{grad} q)=\int_{\Omega} \boldsymbol{g} \cdot \operatorname{grad} q \mathrm{~d} \boldsymbol{x}-b_{D}(\alpha \operatorname{grad} q, p) .
$$

It follows from the formula $b_{D}(\alpha \operatorname{grad} q, p)=a_{L}(p, q)$ combined with (2.4) that

$$
\forall q \in H^{1}(\Omega), \quad b_{D}(\boldsymbol{u}, q)=-\int_{\Omega}(f q)(\boldsymbol{x}) \mathrm{d} \boldsymbol{x},
$$

so that $(\boldsymbol{u}, p)$ is a solution of (2.10). The uniqueness of $\boldsymbol{u}$ is also a consequence of the Lax-Milgram lemma.

2) Conversely, let $(\boldsymbol{u}, p)$ be a solution of (2.10). Taking $\boldsymbol{v}$ equal to $\alpha \operatorname{grad} q$ in the first line of (2.10) and using the second line of (2.10) together with the same arguments as previously yields that $p$ is a solution of (2.4).

To conclude, we recall some regularity properties of the solution of problems (2.4) and (2.10). The proof of the next lemma is based on an idea of Meyers [16] (see also [13]) and is explicitly carried out in [7], Proposition 2.2 for the Laplace equation (1.2).

Proposition 2.5. There exists a constant $c_{\Omega}$ depending only on the geometry of $\Omega$ such that, for all data $(f, \boldsymbol{g})$ satisfying (2.3), the solution $p$ of problem (2.4) belongs to $H^{s+1}(\Omega)$ and the solution $(\boldsymbol{u}, p)$ of problem (2.10) belongs to $H^{s}(\Omega)^{2} \times H^{s+1}(\Omega)$, for all real numbers $s<s_{0}$, where $s_{0}$ is given by

$$
s_{0}=\min \left\{\frac{1}{2}, c_{\Omega}\left|\log \left(1-\frac{\alpha_{\min }}{\alpha_{\max }}\right)\right|\right\} .
$$

Clearly, it can be shown that the solution is more regular locally. For instance, if the data $f$ and $\operatorname{div} \boldsymbol{g}$ belong to $H^{1}(\Omega)$ and for a fixed $\lambda>0$, the solution $p$ of problem (2.4) belongs to $H^{s+1}\left(\Omega^{*}\right)$ and the solution $(\boldsymbol{u}, p)$ of problem (2.10) belongs to $H^{s}\left(\Omega^{*}\right)^{2} \times H^{s+1}\left(\Omega^{*}\right)$ for all $s<2$ and all $\Omega^{*}$ such that

$$
\left.\bar{\Omega}^{*} \subset\right]-1,1[\times] 1-\varepsilon+\lambda, 1\left[\text { or } \bar{\Omega}^{*} \subset\right]-1,-\lambda[\times]-1,1-\varepsilon-\lambda\left[\text { or } \bar{\Omega}^{*} \subset\right] \lambda, 1[\times]-1,1-\varepsilon-\lambda[.
$$


However, the investigation of the regularity of the solutions in a neighbourhood of the discontinuities of $\alpha$ is a much more complex task.

Finally, it must be noted that all the previous regularity properties are independent of $\varepsilon$. However, the norms of the solutions in the corresponding Sobolev spaces depend on $\varepsilon$, as can be checked by using the one-dimensional homothety which maps the rectangle $\Omega_{1}$ onto the square $]-1,1[\times] 1-\varepsilon, 3-\varepsilon[$.

\section{The DISCRETE PROBLEMS AND THEIR WELL-POSEDNESS}

The skeleton $\mathcal{S}$ of the decomposition, which is equal to $\cup_{k=1}^{3} \partial \Omega_{k} \backslash \partial \Omega$, admits a decomposition without overlap into mortars

$$
\overline{\mathcal{S}}=\bigcup_{m=1}^{M^{-}} \bar{\gamma}_{m}^{-} \quad \text { and } \quad \gamma_{m}^{-} \cap \gamma_{m^{\prime}}^{-}=\emptyset, \quad 1 \leq m<m^{\prime} \leq M^{-}
$$

where each $\gamma_{m}^{-}$is a whole edge of one of the $\Omega_{k}$, which is then denoted by $\Omega_{m}^{-}$. Note that the choice of this decomposition is not unique. However it is decided a priori for all the discretizations we consider. Once it is fixed, we have another decomposition of the skeleton into non-mortars

$$
\overline{\mathcal{S}}=\bigcup_{m=1}^{M^{+}} \bar{\gamma}_{m}^{+} \quad \text { and } \quad \gamma_{m}^{+} \cap \gamma_{m^{\prime}}^{+}=\emptyset, \quad 1 \leq m<m^{\prime} \leq M^{+}
$$

where each $\gamma_{m}^{+}$is a whole edge of one of the $\Omega_{k}$, here denoted by $\Omega_{m}^{+}$, and either $\gamma_{m}^{+}$does not coincide with any $\gamma_{m^{\prime}}^{-}$or, if $\gamma_{m}^{+}$is equal to a $\gamma_{m^{\prime}}^{-}, \Omega_{m}^{+}$is different from $\Omega_{m^{\prime}}^{-}$.

To illustrate this rather abstract definition, we observe that, for the geometry defined in (1.1) and shown in Figure 1, four choices of mortars (hence of non-mortars) are possible, as described in Figure 2. The skeleton is the union of the vertical segment $\{0\} \times]-1,1-\varepsilon[$ and the horizontal segment $]-1,1[\times\{1-\varepsilon\}$. Thus,

- the vertical segment $\{0\} \times]-1,1-\varepsilon\left[\right.$ is both a mortar $\gamma_{1}^{-}$and a non-mortar $\gamma_{1}^{+}$but $\Omega_{1}^{-}$can be taken equal to $\Omega_{2}$ (then, $\Omega_{1}^{+}$is equal to $\Omega_{3}$ ) or to $\Omega_{3}$ (then, $\Omega_{1}^{+}$is equal to $\Omega_{2}$ ),

- the horizontal segment $]-1,1\left[\times\{1-\varepsilon\}\right.$ is either one mortar $\gamma_{2}^{-}$, so that $\Omega_{2}^{-}$is equal to $\Omega_{1}$ and

$$
\left.\gamma_{2}^{+}=\right]-1,0\left[\times\{1-\varepsilon\}, \quad \Omega_{2}^{+}=\Omega_{2} \quad \text { and } \quad \gamma_{3}^{+}=\right] 0,1\left[\times\{1-\varepsilon\}, \quad \Omega_{3}^{+}=\Omega_{3},\right.
$$

or divided into two mortars $\gamma_{2}^{-}$and $\gamma_{3}^{-}$, with

$$
\left.\gamma_{2}^{-}=\right]-1,0\left[\times\{1-\varepsilon\}, \quad \Omega_{2}^{-}=\Omega_{2} \quad \text { and } \quad \gamma_{3}^{-}=\right] 0,1\left[\times\{1-\varepsilon\}, \quad \Omega_{3}^{-}=\Omega_{3},\right.
$$

so that $\gamma_{2}^{+}$is equal to $]-1,1\left[\times\{1-\varepsilon\}\right.$ and $\Omega_{2}^{+}$to $\Omega_{1}$.

In all cases, both $M^{-}$and $M^{+}$are equal to 2 or 3 , and their sum is always 5 .

In order to take into account the large aspect ratio of the domain $\Omega_{1}$, the discretization parameter $\delta$ is here a 4 -tuple $\left(M_{1}, N_{1}, N_{2}, N_{3}\right)$ of integers $\geq 2$. Indeed, the local discrete spaces are defined as follows:

- The space $X_{\delta}^{1}$ is the space of restrictions to $\Omega_{1}$ of polynomials of degree $\leq N_{1}$ with respect to $x$ and of degree $\leq M_{1}$ with respect to $y$;

- For $k=2$ and 3 , the space $X_{\delta}^{k}$ is the space of restrictions to $\Omega_{k}$ of polynomials of degree $\leq N_{k}$ with respect to both $x$ and $y$.

For each $\gamma_{m}^{+}$, we denote by $N_{m}^{+}$the integer $N_{k}$, where $k$ is such that $\Omega_{m}^{+}$is equal to $\Omega_{k}$, and, for any nonnegative integer $N$, by $\mathbb{P}_{N}\left(\gamma_{m}^{+}\right)$the space of restrictions to $\gamma_{m}^{+}$of polynomials with one variable and degree $\leq N$. 

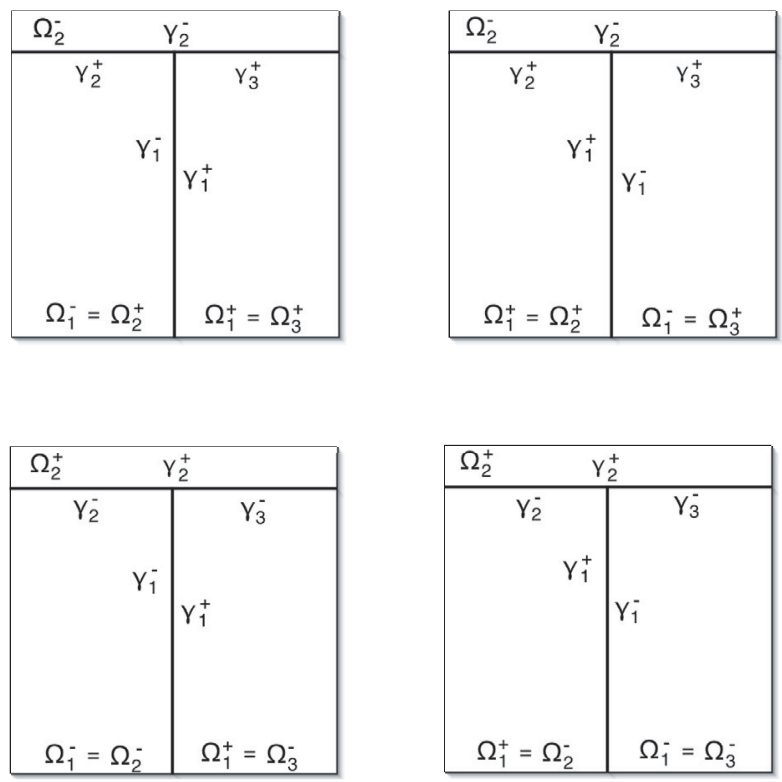

Figure 2. The four possible choices of mortars and non-mortars.

The mortar discrete space $\mathbb{X}_{\delta}$ is then defined in the usual way, according to [9] (see also [11] for a more recent description). It is the space of functions $q_{\delta}$ such that:

- the restriction $q_{\delta \mid \Omega_{k}}$ to each $\Omega_{k}, 1 \leq k \leq 3$, belongs to $X_{\delta}^{k}$;

- the following matching condition holds for all $m, 1 \leq m \leq M^{+}$,

$$
\forall \psi_{\delta} \in \mathbb{P}_{N_{m}^{+}-2}\left(\gamma_{m}^{+}\right), \quad \int_{\gamma_{m}^{+}}\left(q_{\delta \mid \Omega_{m}^{+}}-\varphi\left(q_{\delta}\right)\right)(\tau) \psi_{\delta}(\tau) \mathrm{d} \tau=0,
$$

where the mortar function $\varphi\left(q_{\delta}\right)$ associated with $q_{\delta}$ is defined on each $\gamma_{m}^{-}, 1 \leq m \leq M^{-}$, as the trace of $q_{\delta} \mid \Omega_{m}^{-}$. Note that the space $\mathbb{X}_{\delta}$ is not contained in $H^{1}(\Omega)$, which means that the discretization is not conforming.

Let $\mathbb{P}_{N}(-1,1)$ denote the space of restrictions to ] $-1,1[$ of polynomials in one variable and degree $\leq N$. The Gauss-Lobatto formula on the interval ] - 1,1[ can be written as follows: For each positive integer $N$, with the notation $\xi_{0}^{N}=-1$ and $\xi_{N}^{N}=1$, there exists a unique set of nodes $\xi_{j}^{N}, 1 \leq j \leq N-1$, and weights $\rho_{j}$, $0 \leq j \leq N$, such that

$$
\forall \Phi \in \mathbb{P}_{2 N-1}(-1,1), \quad \int_{-1}^{1} \Phi(\zeta) \mathrm{d} \zeta=\sum_{j=0}^{N} \Phi\left(\xi_{j}^{N}\right) \rho_{j}^{N}
$$

where the $\xi_{j}^{N}$ are equal to the zeros of the first derivative of the Legendre polynomial of degree $N$ and the $\rho_{j}^{N}$ are positive. Moreover, the additional positivity property holds

$$
\forall \varphi_{N} \in \mathbb{P}_{N}(-1,1), \quad\left\|\varphi_{N}\right\|_{L^{2}(-1,1)}^{2} \leq \sum_{j=0}^{N} \varphi_{N}^{2}\left(\xi_{j}^{N}\right) \rho_{j}^{N} \leq 3\left\|\varphi_{N}\right\|_{L^{2}(-1,1)}^{2}
$$


Next,

- on $\Omega_{1}$, we first take $N$ equal to $N_{1}$ and, by homothety and translation, we construct from the $\xi_{j}^{N_{1}}$ and $\rho_{j}^{N_{1}}, 0 \leq j \leq N_{1}$, the nodes and weights $\xi_{1 j}^{(x)}$ and $\rho_{1 j}^{(x)}$ in the $x$-direction. On the other hand, we take $N$ equal to $M_{1}$ and, by homothety and translation, we construct from the $\xi_{j}^{M_{1}}$ and $\rho_{j}^{M_{1}}, 0 \leq j \leq M_{1}$, the nodes and weights $\xi_{1 j}^{(y)}$ and $\rho_{1 j}^{(y)}$ in the $y$-direction;

- for $k=2$ and 3 , we take $N$ equal to $N_{k}$ and, by homothety and translation, we construct from the $\xi_{j}^{N_{k}}$ and $\rho_{j}^{N_{k}}, 0 \leq j \leq N_{k}$, the nodes and weights $\xi_{k j}^{(x)}$ and $\rho_{k j}^{(x)}$, resp. $\xi_{k j}^{(y)}$ and $\rho_{k j}^{(y)}$, in the $x$-direction, resp. in the $y$-direction.

This leads to a discrete product, defined on all functions $u$ and $v$ which have continuous restrictions to all $\bar{\Omega}_{k}$, $1 \leq k \leq K$ :

$$
\begin{aligned}
&((u, v))_{\delta}=\sum_{k=1}^{3}((u, v))_{\delta}^{k} \quad \text { with } \quad((u, v))_{\delta}^{1}=\sum_{i=0}^{N_{1}} \sum_{j=0}^{M_{1}} u\left(\xi_{1 i}^{(x)}, \xi_{1 j}^{(y)}\right) v\left(\xi_{1 i}^{(x)}, \xi_{1 j}^{(y)}\right) \rho_{1 i}^{(x)} \rho_{1 j}^{(y)} \\
& \text { and } \quad((u, v))_{\delta}^{k}=\sum_{i=0}^{N_{k}} \sum_{j=0}^{N_{k}} u\left(\xi_{k i}^{(x)}, \xi_{k j}^{(y)}\right) v\left(\xi_{k i}^{(x)}, \xi_{k j}^{(y)}\right) \rho_{k i}^{(x)} \rho_{k j}^{(y)}, \quad k=2,3 .
\end{aligned}
$$

The exactness property of this product follows from (3.6). Let also $\mathcal{I}_{\delta}^{k}, 1 \leq k \leq K$, denote the Lagrange interpolation operators at all nodes $\left(\xi_{k i}^{(x)}, \xi_{k j}^{(y)}\right)$ with values in $X_{\delta}^{k}$, and $\mathcal{I}_{\delta}$ the operator such that its restriction to each $\Omega_{k}$ coincides with $\mathcal{I}_{\delta}^{k}$.

We are now in a position to present the discrete problem associated with problem (2.4). We assume that the functions $f$ and $\boldsymbol{g}$ have continuous restrictions to all $\bar{\Omega}_{k}, 1 \leq k \leq 3$. Then, the discrete problem can be written as:

Find $p_{\delta}$ in $\mathbb{X}_{\delta} \cap L_{0}^{2}(\Omega)$ such that

$$
\forall q_{\delta} \in \mathbb{X}_{\delta}, \quad a_{L \delta}\left(p_{\delta}, q_{\delta}\right)=\left(\left(f_{\delta}, q_{\delta}\right)\right)_{\delta}+\left(\left(\boldsymbol{g}, \operatorname{grad} q_{\delta}\right)\right)_{\delta},
$$

where the bilinear form $a_{L \delta}(\cdot, \cdot)$ is defined by

$$
a_{L \delta}\left(p_{\delta}, q_{\delta}\right)=\sum_{k=1}^{3} \alpha_{k}\left(\left(\operatorname{grad} p_{\delta}, \operatorname{grad} q_{\delta}\right)\right)_{\delta}^{k},
$$

and the function $f_{\delta}$ is given by

$$
f_{\delta}(x, y)=f(x, y)-\frac{1}{4}((f, 1))_{\delta} .
$$

Indeed, from this last choice, $\left(\left(f_{\delta}, 1\right)\right)_{\delta}$ is equal to zero, so that equation (3.9) can equivalently be enforced for all $q_{\delta}$ in $\mathbb{X}_{\delta}$ or in $\mathbb{X}_{\delta} \cap L_{0}^{2}(\Omega)$.

In order to check the well-posedness of problem (3.9), we need an extension of the Bramble-Hilbert inequality. We introduce the space $\mathbb{X}$ of functions $q$ in $L^{2}(\Omega)$ such that

- the restriction $q_{\mid \Omega_{k}}$ to each $\Omega_{k}, 1 \leq k \leq K$, belongs to $H^{1}\left(\Omega_{k}\right)$,

- the following matching condition holds for all $m, 1 \leq m \leq M^{+}$,

$$
\int_{\gamma_{m}^{+}}\left(q_{\mid \Omega_{m}^{+}}-\varphi(q)\right)(\tau) \mathrm{d} \tau=0
$$

where the mortar function $\varphi(q)$ associated with $q$ is defined on each $\gamma_{m}^{-}, 1 \leq m \leq M^{-}$, as the trace of $q_{\mid \Omega_{m}^{-}}$. The space $\mathbb{X}$ is not a discrete space, and its main advantage is that it contains both $H^{1}(\Omega)$ and all spaces $\mathbb{X}_{\delta}$. 
Lemma 3.1. There exists a constant $c$ such that the following property holds for all functions $q$ in $\mathbb{X} \cap L_{0}^{2}(\Omega)$

$$
\|q\|_{L^{2}(\Omega)} \leq c\left(\sum_{k=1}^{3}|q|_{H^{1}\left(\Omega_{k}\right)}^{2}\right)^{\frac{1}{2}} .
$$

Proof. We note that the norm $q \mapsto\left(\sum_{k=1}^{3}\|q\|_{H^{1}\left(\Omega_{k}\right)}^{2}\right)^{\frac{1}{2}}$ is equivalent to

$$
\|q\|_{L^{2}(\Omega)}+\left(\sum_{k=1}^{3}|q|_{H^{1}\left(\Omega_{k}\right)}^{2}\right)^{\frac{1}{2}}
$$

Moreover, if a function $q$ in $\mathbb{X} \cap L_{0}^{2}(\Omega)$ satisfies $\sum_{k=1}^{3}|q|_{H^{1}\left(\Omega_{k}\right)}^{2}=0$, it is equal to a constant $q_{k}$ in each $\Omega_{k}$. The matching condition on the edge $\left.\gamma_{1}^{+}=\{0\} \times\right]-1,1-\varepsilon\left[\right.$ thus implies that $q_{2}$ and $q_{3}$ are equal. Next, any matching condition on the edge $]-1,1\left[\times\{1-\varepsilon\}\right.$ or part of it yields that $q_{1}$ is equal to $q_{2}=q_{3}$. Therefore, the function $q$ is constant on $\Omega$ and, since it belongs to $L_{0}^{2}(\Omega)$, it is zero. Since the embedding of each $H^{1}\left(\Omega_{k}\right)$ into $L^{2}\left(\Omega_{k}\right)$ is compact, we derive from the Peetre-Tartar lemma, see [14], Chapter I, Theorem 2.1, that the quantities $\left(\sum_{k=1}^{3}\|q\|_{H^{1}\left(\Omega_{k}\right)}^{2}\right)^{\frac{1}{2}}$ and $\left(\sum_{k=1}^{3}|q|_{H^{1}\left(\Omega_{k}\right)}^{2}\right)^{\frac{1}{2}}$ are equivalent in $\mathbb{X} \cap L_{0}^{2}(\Omega)$, whence inequality (3.13).

An immediate consequence of Lemma 3.1 combined with (3.7) is the ellipticity property

$$
\forall q_{\delta} \in \mathbb{X}_{\delta} \cap L_{0}^{2}(\Omega), \quad a_{L \delta}\left(q_{\delta}, q_{\delta}\right) \geq c \alpha_{\min }\left(\sum_{k=1}^{3}\left\|q_{\delta}\right\|_{H^{1}\left(\Omega_{k}\right)}^{2}\right) .
$$

We are thus now in a position to prove the well-posedness of problem (3.9).

Proposition 3.2. For any data $f$ and $\boldsymbol{g}$ which have continuous restrictions to all $\bar{\Omega}_{k}, 1 \leq k \leq 3$, problem (3.9) has a unique solution $p_{\delta}$ in $\mathbb{X}_{\delta} \cap L_{0}^{2}(\Omega)$. Moreover this solution satisfies

$$
\left\|p_{\delta}\right\|_{1, \alpha} \leq c \alpha_{\min }^{-\frac{1}{2}}\left(\left\|\mathcal{I}_{\delta} f\right\|_{L^{2}(\Omega)}+\left\|\mathcal{I}_{\delta} \boldsymbol{g}\right\|_{L^{2}(\Omega)^{2}}\right) .
$$

Proof. From (3.14), the existence and uniqueness of the solution are an immediate consequence of the LaxMilgram lemma. To prove (3.15), we take $q_{\delta}$ equal to $p_{\delta}$ in (3.9) and use (3.7), which gives

$$
\left\|p_{\delta}\right\|_{1, \alpha}^{2} \leq\left(\left(f_{\delta}, p_{\delta}\right)\right)_{\delta}+\left(\left(\boldsymbol{g}, \operatorname{grad} p_{\delta}\right)\right)_{\delta} .
$$

Combining the definition of the operator $\mathcal{I}_{\delta}$ with a Cauchy-Schwarz inequality gives

$$
\left.\left(\left(f_{\delta}, p_{\delta}\right)\right)_{\delta}=\left(\left(\mathcal{I}_{\delta} f_{\delta}, p_{\delta}\right)\right)_{\delta} \leq\left(\left(\mathcal{I}_{\delta} f_{\delta}, \mathcal{I}_{\delta} f_{\delta}\right)\right)_{\delta}^{\frac{1}{2}}\left(p_{\delta}, p_{\delta}\right)\right)_{\delta}^{\frac{1}{2}}
$$

and applying (3.7) in each direction leads to

$$
\left(\left(f_{\delta}, p_{\delta}\right)\right)_{\delta} \leq 9\left\|\mathcal{I}_{\delta} f_{\delta}\right\|_{L^{2}(\Omega)}\left\|p_{\delta}\right\|_{L^{2}(\Omega)} .
$$

Using the definition $(3.11)$ of $f_{\delta}$ together with the identity $((f, 1))_{\delta}=\left(\left(\mathcal{I}_{\delta} f, 1\right)\right)_{\delta}$ and once more Lemma 3.1, we obtain

$$
\left(\left(f_{\delta}, p_{\delta}\right)\right)_{\delta} \leq 9 c \alpha_{\min }^{-\frac{1}{2}}\left(1+\frac{1}{2}\right)\left\|\mathcal{I}_{\delta} f\right\|_{L^{2}(\Omega)}\left\|p_{\delta}\right\|_{1, \alpha} .
$$

Simpler arguments also give

$$
\left(\left(\boldsymbol{g}, \operatorname{grad} p_{\delta}\right)\right)_{\delta} \leq 3 \alpha_{\min }^{-\frac{1}{2}}\left\|\mathcal{I}_{\delta} \boldsymbol{g}\right\|_{L^{2}(\Omega)^{2}}\left\|p_{\delta}\right\|_{1, \alpha}
$$


Combining all this yields the desired estimate.

We now consider the discrete problem associated with problem (2.10). We introduce the space $\mathbb{Y}_{\delta}$ of functions such that their restrictions to each $\Omega_{k}, 1 \leq k \leq 3$, belong to $X_{\delta}^{k}$. Here also, we assume that the functions $f$ and $\boldsymbol{g}$ have continuous restrictions to all $\bar{\Omega}_{k}, 1 \leq k \leq 3$. Then, the discrete problem may be written as:

Find $\left(\boldsymbol{u}_{\delta}, p_{\delta}\right.$ in $\mathbb{Y}_{\delta}^{2} \times\left(\mathbb{X}_{\delta} \cap L_{0}^{2}(\Omega)\right)$ such that

$$
\begin{aligned}
& \forall \boldsymbol{v}_{\delta} \in \mathbb{Y}_{\delta}^{2}, \quad a_{D \delta}\left(\boldsymbol{u}_{\delta}, \boldsymbol{v}_{\delta}\right)+b_{D \delta}\left(\boldsymbol{v}_{\delta}, p_{\delta}\right)=\sum_{k=1}^{3} \alpha_{k}^{-1}\left(\left(\boldsymbol{g}, \boldsymbol{v}_{\delta}\right)\right)_{\delta}^{k} \\
& \forall q_{\delta} \in \mathbb{X}_{\delta}, \quad b_{D \delta}\left(\boldsymbol{u}_{\delta}, q_{\delta}\right)=-\left(\left(f_{\delta}, q_{\delta}\right)\right)_{\delta},
\end{aligned}
$$

where the function $f_{\delta}$ is introduced in $(3.11)$ and the bilinear forms $a_{D \delta}(\cdot, \cdot)$ and $b_{D \delta}(\cdot, \cdot)$ are defined by

$$
a_{D \delta}\left(\boldsymbol{u}_{\delta}, \boldsymbol{v}_{\delta}\right)=\sum_{k=1}^{3} \alpha_{k}^{-1}\left(\left(\boldsymbol{u}_{\delta}, \boldsymbol{v}_{\delta}\right)\right)_{\delta}^{k}, \quad b_{D \delta}\left(\boldsymbol{v}_{\delta}, q_{\delta}\right)=\left(\left(\boldsymbol{v}_{\delta}, \operatorname{grad} q_{\delta}\right)\right)_{\delta} .
$$

The ellipticity of the form $a_{D \delta}(\cdot, \cdot)$ on $\mathbb{Y}_{\delta}^{2}$ is an easy consequence of $(3.7)$ :

$$
\forall \boldsymbol{v}_{\delta} \in \mathbb{Y}_{\delta}^{2}, \quad a_{D \delta}\left(\boldsymbol{v}_{\delta}, \boldsymbol{v}_{\delta}\right) \geq\left\|\boldsymbol{v}_{\delta}\right\|_{0, \alpha^{-1}}^{2}
$$

The same argument as in the continuous case leads to an inf-sup condition on the form $b_{D \delta}(\cdot, \cdot)$.

Lemma 3.3. The following inf-sup condition holds

$$
\forall q_{\delta} \in \mathbb{X}_{\delta} \cap L_{0}^{2}(\Omega), \quad \sup _{\boldsymbol{v}_{\delta} \in \mathbb{Y}_{\delta}^{2}} \frac{b_{D \delta}\left(\boldsymbol{v}_{\delta}, q_{\delta}\right)}{\left\|\boldsymbol{v}_{\delta}\right\|_{0, \alpha^{-1}}} \geq\left\|q_{\delta}\right\|_{1, \alpha}
$$

Proof. It is readily checked that, for any $q_{\delta}$ in $\mathbb{X}_{\delta}$, the functions $\operatorname{grad} q_{\delta}$ and $\alpha \operatorname{grad} q_{\delta}$ belong to $\mathbb{Y}_{\delta}^{2}$. Thus, by taking $\boldsymbol{v}_{\delta}$ equal to $\alpha \operatorname{grad} q_{\delta}$, we obtain

$$
b_{D \delta}\left(\boldsymbol{v}_{\delta}, q_{\delta}\right)=\sum_{k=1}^{3} \alpha_{k}\left(\left(\operatorname{grad} q_{\delta}, \operatorname{grad} q_{\delta}\right)\right)_{\delta}^{k} \quad \text { and } \quad\left\|\boldsymbol{v}_{\delta}\right\|_{0, \alpha^{-1}}=\left\|q_{\delta}\right\|_{1, \alpha}
$$

Using once more (3.7) in the first equality leads to

$$
b_{D \delta}\left(\boldsymbol{v}_{\delta}, q_{\delta}\right) \geq\left\|q_{\delta}\right\|_{1, \alpha}^{2}
$$

Combining all this gives the desired condition.

We state and prove the well-posedness of problem (3.16). The proof involves the discrete kernel

$$
V_{\delta}=\left\{\boldsymbol{v}_{\delta} \in \mathbb{Y}_{\delta}^{2} ; \forall q_{\delta} \in \mathbb{X}_{\delta}, b_{D \delta}\left(\boldsymbol{v}_{\delta}, q_{\delta}\right)=0\right\}
$$

Proposition 3.4. For any data $f$ and $\boldsymbol{g}$ which have continuous restrictions to all $\bar{\Omega}_{k}, 1 \leq k \leq 3$, problem (3.16) has a unique solution $\left(\boldsymbol{u}_{\delta}, p_{\delta}\right)$ in $\mathbb{Y}_{\delta}^{2} \times\left(\mathbb{X}_{\delta} \cap L_{0}^{2}(\Omega)\right)$. Moreover, this solution satisfies

$$
\left\|\boldsymbol{u}_{\delta}\right\|_{0, \alpha^{-1}}+\left\|p_{\delta}\right\|_{1, \alpha} \leq c \alpha_{\min }^{-\frac{1}{2}}\left(\left\|\mathcal{I}_{\delta} f\right\|_{L^{2}(\Omega)}+\left\|\mathcal{I}_{\delta} \boldsymbol{g}\right\|_{L^{2}(\Omega)^{2}}\right) .
$$


Proof. Let us first take $f$ and $\boldsymbol{g}$ equal to zero in (3.16). Choosing $\boldsymbol{v}_{\delta}$ equal to $\boldsymbol{u}_{\delta}$ in the first line of this equation yields that $a_{D \delta}\left(\boldsymbol{u}_{\delta}, \boldsymbol{u}_{\delta}\right)$ is zero. It therefore follows from (3.18) that $\boldsymbol{u}_{\delta}$ is zero. We now have

$$
\forall \boldsymbol{v}_{\delta} \in \mathbb{Y}_{\delta}^{2}, \quad b_{D \delta}\left(\boldsymbol{v}_{\delta}, p_{\delta}\right)=0
$$

hence $p_{\delta}$ is also zero from (3.19). On the other hand, problem (3.16) results into a square linear system since, thanks to the choice of $f_{\delta}$, the second line in (3.16) can equivalently be enforced for all $q_{\delta}$ in $\mathbb{X}_{\delta}$ or in $\mathbb{X}_{\delta} \cap L_{0}^{2}(\Omega)$. The existence and uniqueness of the solution $\left(\boldsymbol{u}_{\delta}, p_{\delta}\right)$ are a consequence of the previous arguments. We now prove estimate (3.21) by following the same steps as in the proof of Proposition 2.2.

1) It follows from the inf-sup condition (3.19) that there exists a function $\boldsymbol{u}_{\delta}^{*}$ in $\mathbb{Y}_{\delta}^{2}$ such that

$\forall q_{\delta} \in \mathbb{X}_{\delta} \cap L_{0}^{2}(\Omega), \quad b_{D \delta}\left(\boldsymbol{u}_{\delta}^{*}, q_{\delta}\right)=-\left(\left(f_{\delta}, q_{\delta}\right)\right)_{\delta}$

$$
\text { and }\left\|\boldsymbol{u}_{\delta}^{*}\right\|_{0, \alpha^{-1}} \leq c \alpha_{\min }^{-\frac{1}{2}}\left\|\mathcal{I}_{\delta} f_{\delta}\right\|_{L^{2}(\Omega)} \leq \frac{3 c}{2} \alpha_{\min }^{-\frac{1}{2}}\left\|\mathcal{I}_{\delta} f\right\|_{L^{2}(\Omega)} .
$$

2) Because of the choice of $\boldsymbol{u}_{\delta}^{*}$, the function $\boldsymbol{u}_{\delta}^{0}=\boldsymbol{u}_{\delta}-\boldsymbol{u}_{\delta}^{*}$ belongs to $V_{\delta}$ and satisfies

$$
\forall \boldsymbol{v}_{\delta} \in V_{\delta}, \quad a_{D \delta}\left(\boldsymbol{u}_{\delta}^{0}, \boldsymbol{v}_{\delta}\right)=\sum_{k=1}^{3} \alpha_{k}^{-1}((\boldsymbol{g}, \boldsymbol{v}))_{\delta}^{k}-a_{D \delta}\left(\boldsymbol{u}_{\delta}^{*}, \boldsymbol{v}_{\delta}\right)
$$

We thus derive from (3.18) and (3.7) that

$$
\left\|\boldsymbol{u}_{\delta}^{0}\right\|_{0, \alpha^{-1}} \leq c\left(\alpha_{\min }^{-\frac{1}{2}}\left\|\mathcal{I}_{\delta} \boldsymbol{g}\right\|_{L^{2}(\Omega)^{2}}+\left\|\boldsymbol{u}_{\delta}^{*}\right\|_{0, \alpha^{-1}}\right) .
$$

3) The pressure $p_{\delta}$ satisfies

$$
\forall \boldsymbol{v}_{\delta} \in \mathbb{Y}_{\delta}^{2}, \quad b_{D \delta}\left(\boldsymbol{v}_{\delta}, p_{\delta}\right)=\sum_{k=1}^{3} \alpha_{k}^{-1}\left(\left(\boldsymbol{g}, \boldsymbol{v}_{\delta}\right)\right)_{\delta}^{k}-a_{D \delta}\left(\boldsymbol{u}_{\delta}^{0}+\boldsymbol{u}_{\delta}^{*}, \boldsymbol{v}_{\delta}\right) .
$$

Applying the inf-sup condition (3.19) once more yields

$$
\left\|p_{\delta}\right\|_{1, \alpha} \leq c\left(\alpha_{\min }^{-\frac{1}{2}}\left\|\mathcal{I}_{\delta} \boldsymbol{g}\right\|_{L^{2}(\Omega)^{2}}+\left\|\boldsymbol{u}_{\delta}^{0}\right\|_{0, \alpha^{-1}}+\left\|\boldsymbol{u}_{\delta}^{*}\right\|_{0, \alpha^{-1}}\right) .
$$

Combining (3.22), (3.24) and (3.25) yields estimate (3.21).

As for the continuous problems, we now compare the solutions of problems (3.9) and (3.16).

Proposition 3.5. Problems (3.9) and (3.16) are equivalent in the following sense:

(i) For any solution $p_{\delta}$ of problem (3.9), there exists a unique function $\boldsymbol{u}_{\delta}$ in $\mathbb{Y}_{\delta}^{2}$ such that the pair $\left(\boldsymbol{u}_{\delta}, p_{\delta}\right)$ is a solution of problem (3.16).

(ii) For any solution $\left(\boldsymbol{u}_{\delta}, p_{\delta}\right)$ of problem (3.16), the function $p_{\delta}$ is a solution of problem (3.9).

Proof. Let $\left(\boldsymbol{u}_{\delta}, p_{\delta}\right)$ be a solution of problem (3.16). By noting that $\boldsymbol{u}_{\delta}+\alpha \operatorname{grad} p_{\delta}-\mathcal{I}_{\delta} \boldsymbol{g}$ belongs to $\mathbb{Y}_{\delta}^{2}$ and choosing $\boldsymbol{v}_{\delta}$ equal to this function in the first line of (3.16) implies that this line can equivalently be written

$$
\boldsymbol{u}_{\delta}+\alpha \operatorname{grad} p_{\delta}=\mathcal{I}_{\delta} \boldsymbol{g} \quad \text { in } \Omega .
$$

Inserting this equality in the second line of (3.16) gives that $p_{\delta}$ is a solution of (3.9). Conversely, for any solution $p_{\delta}$ of problem (3.9), defining $\boldsymbol{u}_{\delta}$ by (3.26) yields that $\left(\boldsymbol{u}_{\delta}, p_{\delta}\right)$ is a solution of (3.16). The uniqueness of 
$\boldsymbol{u}_{\delta}$ is a consequence of (3.18).

Consequently, problems (3.9) and (3.16) lead to the same discrete solution $p_{\delta}$. However, as will become apparent later, their implementation is rather different.

\section{ERror estimates}

We are now interested in establishing an upper bound for the error issued from the discretization of problem (2.4). Due to the nonconformity of the method, we multiply the first line of problem (1.2) by a function $r_{\delta}$ in $\mathbb{X}_{\delta}$ and integrate by parts on each $\Omega_{k}$. This gives

$$
a_{L}\left(p, r_{\delta}\right)=\int_{\Omega}\left(f r_{\delta}+\boldsymbol{g} \cdot \operatorname{grad} r_{\delta}\right)(\boldsymbol{x}) \mathrm{d} \boldsymbol{x}-\int_{\mathcal{S}}\left(\alpha \partial_{n} p-\boldsymbol{g} \cdot \boldsymbol{n}\right)(\tau)\left[r_{\delta}(\tau)\right] \mathrm{d} \tau,
$$

where, if at each point $\tau$ of $\mathcal{S}, \boldsymbol{n}$ denotes the unit outward normal vector directed from an $\Omega_{k}$ towards an $\Omega_{k^{\prime}}$, $\left[r_{\delta}(\tau)\right]$ stands for the jump $r_{\delta \mid \Omega_{k^{\prime}}}(\tau)-r_{\delta \mid \Omega_{k}}(\tau)$.

Let now $q_{\delta}$ be any function in $\mathbb{X}_{\delta}$. By using (3.7) once more, we have

$$
\left\|p_{\delta}-q_{\delta}\right\|_{1, \alpha}^{2} \leq a_{L \delta}\left(p_{\delta}-q_{\delta}, p_{\delta}-q_{\delta}\right)
$$

Adding and subtracting (4.1) and (3.9) yield

$$
\begin{aligned}
\left\|p_{\delta}-q_{\delta}\right\|_{1, \alpha}^{2} \leq a_{L}(p- & \left.q_{\delta}, p_{\delta}-q_{\delta}\right)+\int_{\mathcal{S}}\left(\alpha \partial_{n} p-\boldsymbol{g} \cdot \boldsymbol{n}\right)(\tau)\left[\left(p_{\delta}-q_{\delta}\right)(\tau)\right] \mathrm{d} \tau \\
& +\left(a_{L}-a_{L \delta}\right)\left(q_{\delta}, p_{\delta}-q_{\delta}\right)+\left(\left(f_{\delta}, p_{\delta}-q_{\delta}\right)\right)_{\delta}-\int_{\Omega} f(\boldsymbol{x})\left(p_{\delta}-q_{\delta}\right)(\boldsymbol{x}) \mathrm{d} \boldsymbol{x} \\
& +\left(\left(\boldsymbol{g}, \operatorname{grad}\left(p_{\delta}-q_{\delta}\right)\right)\right)_{\delta}-\int_{\Omega}\left(\boldsymbol{g} \cdot \operatorname{grad}\left(p_{\delta}-q_{\delta}\right)\right)(\boldsymbol{x}) \mathrm{d} \boldsymbol{x} .
\end{aligned}
$$

Using the Cauchy-Schwarz inequality several times, and subsequently a triangle inequality, we obtain

$$
\left\|p-p_{\delta}\right\|_{1, \alpha} \leq c\left(\left\|p-q_{\delta}\right\|_{1, \alpha}+\sup _{r_{\delta} \in \mathbb{X}_{\delta}} \frac{\int_{\mathcal{S}}\left(\alpha \partial_{n} p-\boldsymbol{g} \cdot \boldsymbol{n}\right)(\tau)\left[r_{\delta}(\tau)\right] \mathrm{d} \tau}{\left\|r_{\delta}\right\|_{1, \alpha}}+E_{1}+E_{2}+E_{3}\right)
$$

where the first term in the right-hand side represents the approximation error, the second term is the consistency error and the three terms $E_{1}, E_{2}$ and $E_{3}$ which result from the use of quadrature formulæ are given by

$$
\begin{aligned}
& E_{1}=\sup _{r_{\delta} \in \mathbb{X}_{\delta}} \frac{\left(a_{L}-a_{L \delta}\right)\left(q_{\delta}, r_{\delta}\right)}{\left\|r_{\delta}\right\|_{1, \alpha}}, \\
& E_{2}=\sup _{r_{\delta} \in \mathbb{X}_{\delta}} \frac{\left(\left(f_{\delta}, r_{\delta}\right)\right)_{\delta}-\int_{\Omega} f(\boldsymbol{x}) r_{\delta}(\boldsymbol{x}) \mathrm{d} \boldsymbol{x}}{\left\|r_{\delta}\right\|_{1, \alpha}}, \\
& E_{3}=\sup _{r_{\delta} \in \mathbb{X}_{\delta}} \frac{\left(\left(\boldsymbol{g}, \operatorname{grad} r_{\delta}\right)\right)_{\delta}-\int_{\Omega}\left(\boldsymbol{g} \cdot \operatorname{grad} r_{\delta}\right)(\boldsymbol{x}) \mathrm{d} \boldsymbol{x}}{\left\|r_{\delta}\right\|_{1, \alpha}} .
\end{aligned}
$$

Estimating the terms in $E_{1}, E_{2}$ and $E_{3}$ is easy since each of them involves a sum of local terms on each $\Omega_{k}$. In order to evaluate $E_{1}$, we introduce the orthogonal projection operator $\Pi_{\delta 1}^{k-}$ from $H^{1}\left(\Omega_{k}\right)$ onto $X_{\delta}^{k-}$, where $X_{\delta}^{k-}$ denotes

- the space of restrictions to $\Omega_{1}$ of polynomials of degree $\leq N_{1}-1$ with respect to $x$ and of degree $\leq M_{1}-1$ with respect to $y$ for $k=1$; 
- the space of restrictions to $\Omega_{k}$ of polynomials of degree $\leq N_{k}-1$ with respect to both $x$ and $y$ for $k=2$ and 3 .

Indeed, if $\Pi_{\delta 1}^{-}$denotes the operator equal to $\Pi_{\delta 1}^{k-}$ on each $\Omega_{k}$, the following identity follows from (3.6)

$$
\forall r_{\delta} \in \mathbb{X}_{\delta}, \quad\left(a_{L}-a_{L \delta}\right)\left(\Pi_{\delta 1}^{-} p, r_{\delta}\right)=0
$$

Using this together with a triangle inequality leads to

$$
E_{1} \leq c\left(\left\|p-q_{\delta}\right\|_{1, \alpha}+\sum_{k=1}^{3} \alpha_{k}^{\frac{1}{2}}\left\|p-\Pi_{\delta 1}^{k-} p\right\|_{H^{1}\left(\Omega_{k}\right)}\right) .
$$

To conclude, we recall the approximation properties of the operator $\Pi_{\delta 1}^{k-}$, which are well-known for $k=2$ and 3 , see [5], Theorem 7.3, and are derived from the standard results by using the affine transformation that maps $\Omega_{1}$ onto a square for $k=1$ : For any function $q$ in $H^{s+1}\left(\Omega_{k}\right), s \geq 0$,

$$
\left\|q-\Pi_{\delta 1}^{k-} q\right\|_{H^{1}\left(\Omega_{k}\right)} \leq c \begin{cases}\left(\varepsilon^{s} M_{1}^{-s}+N_{1}^{-s}\right)\|q\|_{H^{s+1}\left(\Omega_{1}\right)} & \text { for } k=1 \\ N_{k}^{-s}\|q\|_{H^{s+1}\left(\Omega_{k}\right)} & \text { for } k=2 \text { and } 3 .\end{cases}
$$

Similarly, let $\Pi_{\delta}^{k}$ denote the orthogonal projection operator from $L^{2}\left(\Omega_{k}\right)$ onto $X_{\delta}^{k-}$. Using the fact that $f$ belongs to $L_{0}^{2}(\Omega)$ and the definition $(3.11)$ of $f_{\delta}$, we have

$$
f-f_{\delta}=\frac{1}{4}\left(\left(\mathcal{I}_{\delta} f, 1\right)\right)_{\delta}=-\frac{1}{4} \int_{\Omega}\left(f-\mathcal{I}_{\delta} f\right)(\boldsymbol{x}) \mathrm{d} \boldsymbol{x} .
$$

Therefore using the same arguments as previously leads to

$$
E_{2} \leq c \sum_{k=1}^{3} \alpha_{k}^{-\frac{1}{2}}\left(\left\|f-\mathcal{I}_{\delta}^{k} f\right\|_{L^{2}\left(\Omega_{k}\right)}+\left\|f-\Pi_{\delta}^{k-} f\right\|_{L^{2}\left(\Omega_{k}\right)}\right),
$$

and

$$
E_{3} \leq c \sum_{k=1}^{3} \alpha_{k}^{-\frac{1}{2}}\left(\left\|\boldsymbol{g}-\mathcal{I}_{\delta}^{k} \boldsymbol{g}\right\|_{L^{2}\left(\Omega_{k}\right)^{2}}+\left\|\boldsymbol{g}-\Pi_{\delta}^{k-} \boldsymbol{g}\right\|_{L^{2}\left(\Omega_{k}\right)^{2}}\right) .
$$

To conclude, we recall from [5], Theorem 7.1, the properties of the operator $\Pi_{\delta}^{k-}$ : For any function $h$ in $H^{\sigma}\left(\Omega_{k}\right)$, $\sigma \geq 0$

$$
\left\|h-\Pi_{\delta}^{k-} h\right\|_{L^{2}\left(\Omega_{k}\right)} \leq c \begin{cases}\left(\varepsilon^{\sigma} M_{1}^{-\sigma}+N_{1}^{-\sigma}\right)\|q\|_{H^{\sigma}\left(\Omega_{1}\right)} & \text { for } k=1, \\ N_{k}^{-\sigma}\|q\|_{H^{\sigma}\left(\Omega_{k}\right)} & \text { for } k=2 \text { and } 3 .\end{cases}
$$

Exactly the same properties hold with the operator $\Pi_{\delta}^{k-}$ replaced by $\mathcal{I}_{\delta}^{k}$ when $\sigma$ is $>1$, see [5], Theorem 14.2 .

We now evaluate the approximation error. We only give an outline of the proof, since the results are standard at least in the isotropic case, see [9], Appendix B. The next statement involves the parameter $\lambda_{\delta}$ defined as $\max \left\{\lambda_{\delta}^{1}, \lambda_{\delta}^{2}\right\}$, with

$$
\lambda_{\delta}^{1}= \begin{cases}\left(\frac{\alpha_{2}}{\alpha_{3}}\right)^{\frac{1}{2}} & \text { if } \quad N_{2} \geq N_{3}, \\ \left(\frac{\alpha_{3}}{\alpha_{2}}\right)^{\frac{1}{2}} & \text { if } \quad N_{3}>N_{2},\end{cases}
$$

and

$$
\lambda_{\delta}^{2}= \begin{cases}\max \left\{\left(\frac{\alpha_{2}}{\alpha_{1}}\right)^{\frac{1}{2}}\left(1+\frac{N_{1}}{N_{2}}\right)^{\frac{1}{2}},\left(\frac{\alpha_{3}}{\alpha_{1}}\right)^{\frac{1}{2}}\left(1+\frac{N_{1}}{N_{3}}\right)^{\frac{1}{2}}\right\} & \text { if }]-1,1\left[\times\{1-\varepsilon\}=\gamma_{2}^{-},\right. \\ \left(1+\frac{N_{1}}{M_{1}}\right) \max \left\{\left(\frac{\alpha_{1}}{\alpha_{2}}\right)^{\frac{1}{2}}\left(1+\frac{N_{2}}{N_{1}}\right)^{\frac{1}{2}},\left(\frac{\alpha_{1}}{\alpha_{3}}\right)^{\frac{1}{2}}\left(1+\frac{N_{3}}{N_{1}}\right)^{\frac{1}{2}}\right\} & \text { if }]-1,1\left[\times\{1-\varepsilon\}=\gamma_{2}^{+} .\right.\end{cases}
$$


Although this definition is rather complex, we prefer to write it explicitly since it allows for optimizing the choice of the mortars as functions of the values of the $\alpha_{k}$.

The proof of the next proposition also requires

- the orthogonal projection operator $\tilde{\pi}_{m}^{+}$from $H_{0}^{1}\left(\gamma_{m}^{+}\right)$onto $\mathbb{P}_{N_{m}^{+}}\left(\gamma_{m}^{+}\right) \cap H_{0}^{1}\left(\gamma_{m}^{+}\right)$for the scalar product associated with the norm $|\cdot|_{H^{1}\left(\gamma_{m}^{+}\right)}$

- a lifting operator $\mathcal{R}_{m}^{+}$from $\mathbb{P}_{N_{m}^{+}}\left(\gamma_{m}^{+}\right) \cap H_{0}^{1}\left(\gamma_{m}^{+}\right)$onto the space of restrictions to $\Omega_{m}^{+}$of polynomials with degree $\leq N_{m}^{+}$with respect to both $x$ and $y$ vanishing on $\partial \Omega_{m}^{+} \backslash \gamma_{m}^{+}$, which satisfies, for all $s$,

$$
\forall \varphi_{\delta} \in \mathbb{P}_{N_{m}^{+}}\left(\gamma_{m}^{+}\right) \cap H_{0}^{1}\left(\gamma_{m}^{+}\right), \quad\left\|\mathcal{R}_{m}^{+} \varphi_{\delta}\right\|_{H^{1}\left(\Omega_{m}^{+}\right)} \leq c\left\|\varphi_{\delta}\right\|_{H_{00}^{\frac{1}{2}}\left(\gamma_{m}^{+}\right)} .
$$

The existence of such an operator is established in [8] (note that its range is not contained in $X_{\delta}^{1}$ when $\Omega_{m}^{+}$is equal to $\Omega_{1}$ and $M_{1}<N_{1}$ ).

Proposition 4.1. Assume that the function $p$ is such that its restriction to each $\Omega_{k}, 1 \leq k \leq K$, belongs to $H^{s_{k}+1}\left(\Omega_{k}\right), s_{k}>\frac{1}{2}$. The following approximation error estimate holds

$$
\inf _{q_{\delta} \in \mathbb{X}_{\delta}}\left\|p-q_{\delta}\right\|_{1, \alpha} \leq c\left(1+\lambda_{\delta}\right)\left(\alpha_{1}^{\frac{1}{2}}\left(\varepsilon^{s_{1}} M_{1}^{-s_{1}}+N_{1}^{-s_{1}}\right)\|q\|_{H^{s_{1}+1}\left(\Omega_{1}\right)}+\sum_{k=2}^{3} \alpha_{k}^{\frac{1}{2}} N_{k}^{-s_{k}}\|q\|_{H^{s_{k}+1}\left(\Omega_{k}\right)}\right) .
$$

Proof. The proof is performed in several steps.

1) We first recall from [5], Theorem 14.2, the following result concerning the interpolation operator

$$
\left\|p-\mathcal{I}_{\delta} p\right\|_{1, \alpha} \leq c\left(\alpha_{1}^{\frac{1}{2}}\left(\varepsilon^{s_{1}} M_{1}^{-s_{1}}+N_{1}^{-s_{1}}\right)\|q\|_{H^{s_{1}+1}\left(\Omega_{1}\right)}+\sum_{k=2}^{3} \alpha_{k}^{\frac{1}{2}} N_{k}^{-s_{k}}\|q\|_{H^{s_{k}+1}\left(\Omega_{k}\right)}\right) .
$$

2) Only when the whole edge $]-1,1\left[\times\{1-\varepsilon\}\right.$ is a mortar, denoted by $\gamma_{2}^{-}$(this is the case in the two top parts of Fig. 2), we define the function

$$
q_{\delta}^{\sharp}= \begin{cases}\left(p-\mathcal{I}_{\delta}^{1} p\right)(\boldsymbol{a})\left(1-x^{2}\right) & \text { on } \Omega_{1}, \\ 0 & \text { on } \Omega_{2} \text { and } \Omega_{3},\end{cases}
$$

where $\boldsymbol{a}$ denotes the corner $(0,1-\varepsilon)$. Thanks to the Gagliardo-Nirenberg inequality, we have

$$
\left\|q_{\delta}^{\sharp}\right\|_{1, \alpha} \leq c \alpha_{1}^{\frac{1}{2}}\left|\left(p-\mathcal{I}_{\delta}^{1} p\right)(\boldsymbol{a})\right| \leq c^{\prime} \alpha_{1}^{\frac{1}{2}}\left\|p-\mathcal{I}_{\delta}^{1} p\right\|_{L^{\infty}\left(\gamma_{2}^{-}\right)} \leq c^{\prime} \alpha_{1}^{\frac{1}{2}}\left\|p-\mathcal{I}_{\delta}^{1} p\right\|_{L^{2}\left(\gamma_{2}^{-}\right)}^{\frac{1}{2}}\left\|p-\mathcal{I}_{\delta}^{1} p\right\|_{H^{1}\left(\gamma_{2}^{-}\right)}^{\frac{1}{2}} .
$$

Since the operator $\mathcal{I}_{\delta}^{1}$ is constructed by tensorization, its restriction to $\gamma_{2}^{-}$is a one-dimensional interpolation operator. From the properties of this operator [5], Theorem 13.4, and the trace theorem, we obtain that

$$
\left\|q_{\delta}^{\sharp}\right\|_{1, \alpha} \leq c \alpha_{1}^{\frac{1}{2}} N_{1}^{-s_{1}}\|q\|_{H^{s_{1}+1}\left(\Omega_{1}\right)} .
$$

Taking $q_{\delta}^{\sharp}$ equal to zero when the edge $]-1,1\left[\times\{1-\varepsilon\}\right.$ is a non-mortar, we observe that the function $q_{\delta}^{\natural}=\mathcal{I}_{\delta} q+q_{\delta}^{\sharp}$ is continuous at all the endpoints of the non-mortars.

3) Finally, we take

$$
q_{\delta}^{b}=\sum_{m=1}^{M^{+}} \mathcal{I}_{\delta} \mathcal{R}_{m}^{+} \tilde{\pi}_{m}^{+}\left(\varphi\left(q_{\delta}^{\natural}\right)-q_{\delta \mid \Omega_{m}^{+}}^{\natural}\right)
$$

where each $\mathcal{R}_{m}^{+}$is extended by zero to $\Omega \backslash \Omega_{m}^{+}$. Note that $\mathcal{I}_{\delta}$ is the identity except when $\Omega_{2}^{+}$is equal to $\Omega_{1}$ and $M_{1}<N_{1}$, where it reduces the degree with respect to $y$ of the image of $\mathcal{R}_{m}^{+}$and also that the previous 
choice can be improved because of the conformity of the bottom part of the decomposition by lifting the jump through $\gamma_{1}^{ \pm}$on the $\Omega_{k}, k=2$ or 3 , corresponding to the largest $N_{k}$ whatever is $\Omega_{1}^{+}$. Rather technical arguments involving a separate treatment of the four possible choices of mortars, give

$$
\left\|q_{\delta}^{b}\right\|_{1, \alpha} \leq c\left(1+\lambda_{\delta}\right)\left(\alpha_{1}^{\frac{1}{2}}\left(\varepsilon^{s_{1}} M_{1}^{-s_{1}}+N_{1}^{-s_{1}}\right)\|q\|_{H^{s_{1}+1}\left(\Omega_{1}\right)}+\sum_{k=2}^{3} \alpha_{k}^{\frac{1}{2}} N_{k}^{-s_{k}}\|q\|_{H^{s_{k}+1}\left(\Omega_{k}\right)}\right) .
$$

To conclude, we set $q_{\delta}=q_{\delta}^{\natural}+q_{\delta}^{b}$. Thus, the jump of $q_{\delta}$ through each $\gamma_{m}^{+}$is equal to $\left(\tilde{\pi}_{m}^{+}-i d\right)\left(\varphi\left(q_{\delta}^{\natural}\right)-q_{\delta \mid \Omega_{m}^{+}}^{\natural}\right)$. Since the mapping: $\chi_{\delta} \mapsto \chi_{\delta}^{\prime \prime}$ is an isomorphism from $\mathbb{P}_{N_{m}^{+}}\left(\gamma_{m}^{+}\right) \cap H_{0}^{1}\left(\gamma_{m}^{+}\right)$onto $\mathbb{P}_{N_{m}^{+}-2}\left(\gamma_{m}^{+}\right)$, the following identity is derived by integration by parts

$$
\forall \varphi \in H_{0}^{1}\left(\gamma_{m}^{+}\right), \forall \psi_{\delta} \in \mathbb{P}_{N_{m}^{+}-2}\left(\gamma_{m}^{+}\right), \quad \int_{\gamma_{m}^{+}}\left(\varphi-\tilde{\pi}_{m}^{+} \varphi\right)(\tau) \psi_{\delta}(\tau) \mathrm{d} \tau=0
$$

so that the function $q_{\delta}$ belongs to $\mathbb{X}_{\delta}$. Estimate (4.12) then follows from (4.13) to (4.15).

It remains to evaluate the consistency error, which is simpler than previously. Let now $\lambda_{\delta}^{3}$ and $\lambda_{\delta}^{4}$ denote the parameters defined by

and

$$
\lambda_{\delta}^{3}= \begin{cases}1 & \text { if }]-1,1\left[\times\{1-\varepsilon\}=\gamma_{2}^{-}\right. \\ \log N_{1} & \text { if }]-1,1\left[\times\{1-\varepsilon\}=\gamma_{2}^{+}\right.\end{cases}
$$

$$
\lambda_{\delta}^{4}=\max _{1 \leq m \leq M+} \max _{k \in \mathcal{E}_{m}^{+}}\left(\frac{\alpha_{m}^{+}}{\alpha_{k}}\right)^{\frac{1}{2}},
$$

where $\mathcal{E}_{m}^{+}$denotes the set of indices $k$ such that $\partial \Omega_{k} \cap \gamma_{m}^{+}$has a positive measure.

Proposition 4.2. Assume that the function $p$ is such that its restriction to each $\Omega_{k}, 1 \leq k \leq K$, belongs to $H^{s_{k}+1}\left(\Omega_{k}\right), s_{k}>\frac{1}{2}$, and also that the data $\boldsymbol{g}$ belong to $H^{\sigma}(\Omega)^{2}, \sigma>\frac{1}{2}$. The following consistency error estimate holds

$$
\begin{aligned}
\sup _{r_{\delta} \in \mathbb{X}_{\delta}} \frac{\int_{\mathcal{S}}\left(\alpha \partial_{n} p-\boldsymbol{g} \cdot \boldsymbol{n}\right)(\tau)\left[r_{\delta}(\tau)\right] \mathrm{d} \tau}{\left\|r_{\delta}\right\|_{1, \alpha}} & \\
& \leq c\left(1+\lambda_{\delta}^{3}\right)\left(1+\lambda_{\delta}^{4}\right) \sum_{k=1}^{3}\left(\alpha_{k}^{\frac{1}{2}} N_{k}^{-s_{k}}\|p\|_{H^{s_{k}+1}\left(\Omega_{k}\right)}+\alpha_{k}^{-\frac{1}{2}} N_{k}^{-\sigma}\|\boldsymbol{g}\|_{H^{\sigma}\left(\Omega_{k}\right)^{2}}\right) .
\end{aligned}
$$

Proof. We use the partition (3.2) of the skeleton into non-mortars. Denoting by $\pi_{m}^{+}$the orthogonal projection operator from $L^{2}\left(\gamma_{m}^{+}\right)$onto $\mathbb{P}_{N_{m}^{+}-2}\left(\gamma_{m}^{+}\right)$, we deduce from the matching conditions (3.5) that

$$
\int_{\gamma_{m}^{+}}\left(\alpha \partial_{n} p-\boldsymbol{g} \cdot \boldsymbol{n}\right)(\tau)\left[r_{\delta}(\tau)\right] \mathrm{d} \tau=\int_{\gamma_{m}^{+}}\left(I d-\pi_{m}^{+}\right)\left(\alpha \partial_{n} p-\boldsymbol{g} \cdot \boldsymbol{n}\right)(\tau)\left[r_{\delta}(\tau)\right] \mathrm{d} \tau .
$$

Using a Cauchy-Schwarz inequality then yields

$$
\int_{\gamma_{m}^{+}}\left(\alpha \partial_{n} p-\boldsymbol{g} \cdot \boldsymbol{n}\right)(\tau)\left[r_{\delta}(\tau)\right] \mathrm{d} \tau \leq\left\|\left(I d-\pi_{m}^{+}\right)\left(\alpha \partial_{n} p-\boldsymbol{g} \cdot \boldsymbol{n}\right)\right\|_{H^{\varepsilon_{m}^{+}-\frac{1}{2}}\left(\gamma_{m}^{+}\right)}\left\|\left[r_{\delta}\right]\right\|_{H^{\frac{1}{2}-\varepsilon_{m}^{+}}\left(\gamma_{m}^{+}\right)}
$$

where all $\varepsilon_{m}^{+}$are taken equal to zero except in the case $m=2$ when the whole edge $]-1,1[\times\{1-\varepsilon\}$ is equal to $\gamma_{2}^{+}$where it satisfies $0<\varepsilon_{2}^{+}<\frac{1}{2}$ (indeed, in this case, the trace of $r_{\delta \mid \bar{\Omega}_{2} \cup \bar{\Omega}_{3}}$ does not belong to $H^{\frac{1}{2}}\left(\gamma_{2}^{+}\right)$for all $r_{\delta}$ in $\mathbb{X}_{\delta}$ since these functions are not necessarily continuous at the point $\left.(0,1-\varepsilon)\right)$. 
Next, we recall from [5], Remark 6.1, that, for any interval $\gamma$, the orthogonal projection operator $\pi_{N}$ from $L^{2}(\gamma)$ onto $\mathbb{P}_{N}(\gamma)$ satisfies, for all nonnegative integers $s$ and $t$ and for any function $\varphi$ in $H^{s}(\gamma)$,

$$
\left\|\varphi-\pi_{N} \varphi\right\|_{H^{-t}(\gamma)} \leq c N^{-t-s}\|\varphi\|_{H^{s}(\gamma)}
$$

Moreover if for instance $0 \leq t \leq \frac{1}{2}$, the constant in the previous inequality is independent of $t$. Applying this result yields the estimate

$$
\begin{aligned}
\int_{\gamma_{m}^{+}}\left(\alpha \partial_{n} p-\boldsymbol{g} \cdot \boldsymbol{n}\right)(\tau) & {\left[r_{\delta}\right](\tau) \mathrm{d} \tau } \\
& \leq c\left(N_{m}^{+}\right)^{\varepsilon_{m}^{+}}\left(\alpha_{m}^{+}\left(N_{m}^{+}\right)^{-s_{m}^{+}}\left\|\partial_{n} p\right\|_{H^{s_{m}^{-}-\frac{1}{2}}\left(\gamma_{m}^{+}\right)}+\left(N_{m}^{+}\right)^{-\sigma}\|\boldsymbol{g}\|_{H^{\sigma-\frac{1}{2}}\left(\gamma_{m}^{+}\right)^{2}}\right)\left\|\left[r_{\delta}\right]\right\|_{H^{\frac{1}{2}-\varepsilon_{m}^{+}}\left(\gamma_{m}^{+}\right)}
\end{aligned}
$$

We conclude by using the trace theorem and also by noting that

1) each $\left(\alpha_{m}^{+}\right)^{\frac{1}{2}}\left\|\left[r_{\delta}\right]\right\|_{H^{\frac{1}{2}-\varepsilon_{m}^{+}}\left(\gamma_{m}^{+}\right)}$is bounded by $\left(\alpha_{m}^{+}\right)^{\frac{1}{2}}$ times the sum of $\left\|r_{\delta}\right\|_{H^{1}\left(\Omega_{m}^{+}\right)}$and of the $\left\|r_{\delta}\right\|_{H^{1}\left(\Omega_{k}\right)}$, $k \in \mathcal{E}_{m}^{+}$, hence by $\left(1+\lambda_{\delta}^{4}\right)$ times $\left\|r_{\delta}\right\|_{1, \alpha}$,

2) when $\varepsilon_{2}^{+}$is not zero, it follows from [12], Remark 2.10, that the mapping: $\left(\varphi_{2}, \varphi_{3}\right) \mapsto \varphi$, where $\varphi$ is equal to $\varphi_{2}$ on $\gamma_{2}^{-}$and to $\varphi_{3}$ on $\gamma_{3}^{-}$is continuous from $\prod_{k=2}^{3} H^{\frac{1}{2}-\varepsilon_{2}^{+}}\left(\gamma_{k}^{-}\right)$into $H^{\frac{1}{2}-\varepsilon_{2}^{+}}\left(\gamma_{2}^{+}\right)$with norm $\leq c\left(\varepsilon_{2}^{+}\right)^{-1}$. Thus taking $\varepsilon_{2}^{+}$equal to $\left(\log N_{1}\right)^{-1}$ leads to the apparition of $\left(1+\lambda_{\delta}^{3}\right)$.

The first error estimate is now derived by inserting into (4.2)

1) estimate (4.12) for the approximation error;

2) estimate (4.18) for the consistency error;

3 ) estimate (4.4) combined with (4.5) and (4.12) for $E_{1}$;

4) estimate (4.6) and (4.7) combined with (4.8) and its analogue for $\mathcal{I}_{\delta}^{k}$ [5], Theorem 14.2, for $E_{2}$ and $E_{3}$.

Theorem 4.3. Assume that the function $p$ is such that its restriction to each $\Omega_{k}, 1 \leq k \leq K$, belongs to $H^{s_{k}+1}\left(\Omega_{k}\right), s_{k}>\frac{1}{2}$, and also that the data $(f, \boldsymbol{g})$ belong to $H^{\sigma}(\Omega) \times H^{\sigma}(\Omega)^{2}, \sigma>1$. The following error estimate holds between the solution $p$ of problem (2.4) and the solution $p_{\delta}$ of problem (3.9)

$$
\left\|p-p_{\delta}\right\|_{1, \alpha} \leq c(E(p)+E(f, \boldsymbol{g}))
$$

where the quantities $E(p)$ and $E(f, \boldsymbol{g})$ are given by

$$
\begin{aligned}
E(p) \leq\left(1+\max \left\{\lambda_{\delta}^{1}, \lambda_{\delta}^{2}\right\}\right)\left(1+\lambda_{\delta}^{3}\right)\left(1+\lambda_{\delta}^{4}\right) & \\
& \left(\alpha_{1}^{\frac{1}{2}}\left(\varepsilon^{s_{1}} M_{1}^{-s_{1}}+N_{1}^{-s_{1}}\right)\|p\|_{H^{s_{1}+1}\left(\Omega_{1}\right)}+\sum_{k=2}^{3} \alpha_{k}^{\frac{1}{2}} N_{k}^{-s_{k}}\|p\|_{H^{s_{k}+1}\left(\Omega_{k}\right)}\right)
\end{aligned}
$$

and

$$
\begin{aligned}
E(f, \boldsymbol{g}) \leq\left(\alpha_{1}^{-\frac{1}{2}}\left(\varepsilon^{\sigma} M_{1}^{-\sigma}+N_{1}^{-\sigma}\right)+\sum_{k=2}^{3} \alpha_{k}^{-\frac{1}{2}} N_{k}^{-\sigma}\right) & \left(\|f\|_{H^{\sigma}(\Omega)}+\left(1+\lambda_{\delta}^{3}\right)\left(1+\lambda_{\delta}^{4}\right)\|\boldsymbol{g}\|_{H^{\sigma}(\Omega)^{2}}\right),
\end{aligned}
$$

for the $\lambda_{\delta}^{j}$ introduced in (4.9), (4.10), (4.16) and (4.17).

By combining Propositions 2.4 and 3.5 with the identity (3.26), we immediately obtain an estimation of the error resulting from the discretization of problem (2.10). 
Theorem 4.4. If the assumptions of Theorem 4.3 are satisfied, the following error estimate holds between the solution $p$ of problem (2.10) and the solution $p_{\delta}$ of problem (3.16)

$$
\left\|\boldsymbol{u}-\boldsymbol{u}_{\delta}\right\|_{0, \alpha^{-1}}+\left\|p-p_{\delta}\right\|_{1, \alpha} \leq c(E(p)+E(f, \boldsymbol{g}))
$$

where the quantities $E(p)$ and $E(f, \boldsymbol{g})$ are defined in (4.20) and (4.21), respectively.

We are interested in the case where the data $(f, \boldsymbol{g})$ are very smooth, so that the quantity $E(f, \boldsymbol{g})$ is much smaller than $E(p)$ and we try to make some further choices in order to optimize $E(p)$. The first natural choice consists in choosing $M_{1}$ such that

$$
c \varepsilon N_{1} \leq M_{1} \leq c^{\prime} \varepsilon N_{1} .
$$

Clearly this reduces the number of degrees of freedom without reducing the accuracy of the discretization. With this choice and in view of (4.10), we take

$$
\left.\gamma_{2}^{-}=\right]-1,1[\times\{1-\varepsilon\}
$$

which apparently improves $\lambda_{\delta}^{2}$ at least when $\varepsilon$ is small. As a consequence, $\lambda_{\delta}^{3}$ is now equal to 1 . When this choice is made, taking all the $N_{k}$ equal to a constant times $\alpha_{k}$ yields that the product $\left(1+\max \left\{\lambda_{\delta}^{1}, \lambda_{\delta}^{2}\right\}\right)\left(1+\lambda_{\delta}^{3}\right)\left(1+\lambda_{\delta}^{4}\right)$ which appears in $E(p)$ behaves like the ratio $\alpha_{\max } / \alpha_{\min }$ in the worst case, which seems unavoidable. To conclude, we note that, in practice, optimal choices of the discretization parameters are not always possible in the numerical simulations. Nevertheless, the previous comments permit an optimization of these choices according to each special situation.

\section{NUMERICAL EXPERIMENTS}

First, we briefly describe the implementation of the two discrete problems. As first proposed in [3], the matching conditions (3.5) are enforced via the introduction of a Lagrange multiplier. More precisely, we define the space

$$
\mathbb{Q}_{\delta}=\prod_{m=1}^{M^{+}} \mathbb{P}_{N_{m}^{+}-2}\left(\gamma_{m}^{+}\right)
$$

Next, we define the bilinear form on $\overline{\mathbb{X}}_{\delta} \times \mathbb{Q}_{\delta}$ by

$$
e\left(q_{\delta}, \mu_{\delta}\right)=\sum_{m=1}^{M^{+}} \int_{\gamma_{m}^{+}}\left(q_{\delta \mid \Omega_{m}^{+}}-\varphi\left(q_{\delta}\right)\right)(\tau) \mu_{m}(\tau) \mathrm{d} \tau, \quad \text { with } \mu_{\delta}=\left(\mu_{1}, \ldots, \mu_{M^{+}}\right)
$$

We denote by $\xi_{m j}^{+}, 0 \leq j \leq N_{m}^{+}$, the nodes of the Gauss-Lobatto quadrature formula on $\Omega_{m}^{+}$which belong to $\bar{\gamma}_{m}^{+}$.

Next, we consider the modified discrete problems

Find $p_{\delta}$ in $\mathbb{Y}_{\delta} \cap L_{0}^{2}(\Omega)$ and $\lambda_{\delta}$ in $\mathbb{Q}_{\delta}$ such that

$$
\begin{array}{ll}
\forall q_{\delta} \in \mathbb{X}_{\delta}, & a_{L \delta}\left(p_{\delta}, q_{\delta}\right)+e\left(q_{\delta}, \lambda_{\delta}\right)=\left(\left(f_{\delta}, q_{\delta}\right)\right)_{\delta}+\left(\left(\boldsymbol{g}, \operatorname{grad} q_{\delta}\right)\right)_{\delta} \\
\forall \mu_{\delta} \in \mathbb{Q}_{\delta}, & e\left(p_{\delta}, \mu_{\delta}\right)=0
\end{array}
$$

and 
Find $\left(\boldsymbol{u}_{\delta}, p_{\delta}\right)$ in $\mathbb{Y}_{\delta}^{2} \times\left(\mathbb{Y}_{\delta} \cap L_{0}^{2}(\Omega)\right)$ and $\lambda_{\delta}$ in $\mathbb{Q}_{\delta}$ such that

$$
\begin{aligned}
& \forall \boldsymbol{v}_{\delta} \in \mathbb{Y}_{\delta}^{2}, \quad a_{D \delta}\left(\boldsymbol{u}_{\delta}, \boldsymbol{v}_{\delta}\right)+b_{D \delta}\left(\boldsymbol{v}_{\delta}, p_{\delta}\right)=\sum_{k=1}^{3} \alpha_{k}^{-1}\left(\left(\boldsymbol{g}, \boldsymbol{v}_{\delta}\right)\right)_{\delta}^{k}, \\
& \forall q_{\delta} \in \mathbb{X}_{\delta}, \quad b_{D \delta}\left(\boldsymbol{u}_{\delta}, q_{\delta}\right)+e\left(q_{\delta}, \lambda_{\delta}\right)=-\left(\left(f_{\delta}, q_{\delta}\right)\right)_{\delta}, \\
& \forall \mu_{\delta} \in \mathbb{Q}_{\delta}, \quad e\left(p_{\delta}, \mu_{\delta}\right)=0 .
\end{aligned}
$$

Their equivalence with problems (3.9) and (3.16), respectively, is proved in [3] in a simpler case.

These new discrete problems can also be written as follows. In problem (5.3), the vector $P$ of unknowns consists of the $p_{\delta}\left(\xi_{k i}^{(x)}, \xi_{k j}^{(y)}\right)$ minus the value at one node which is usually taken equal to zero (the fact that $p_{\delta}$ belongs to $L_{0}^{2}(\Omega)$ is recovered in a post-processing step). If this node belongs to $\Omega_{1}$ for instance, $P$ consists of three blocks of sizes $\left(M_{1}+1\right)\left(N_{1}+1\right)-1$ and $\left(N_{k}+1\right)^{2}, k=2,3$, respectively. The vector $\Lambda$ of auxiliary unknowns is made of the $\lambda_{\delta}\left(\xi_{m j}^{+}\right), 1 \leq j \leq N_{m}^{+}-1$, so it consists of $M^{+}$blocks. Problem (5.3) is equivalent to the linear system

$$
\left(\begin{array}{ll}
A_{L} & E^{T} \\
E & 0
\end{array}\right)\left(\begin{array}{l}
P \\
\Lambda
\end{array}\right)\left(\begin{array}{l}
H \\
0
\end{array}\right)
$$

where the vector $H$ depends on the values of $(f, \boldsymbol{g})$ at the Gauss-Lobatto nodes. The main advantage of the system resulting from this approach is that the matrix $A_{L}$ is block-diagonal, made of one block per $\Omega_{k}$, while the size of the matrix $E$ (or at least the number of non-zero coefficients in it) is much smaller. Moreover, since the matrix $A_{L}$ is symmetric, the global matrix is also symmetric.

Similarly, let $U$ be the vector consisting of the $u_{\delta}\left(\xi_{k i}^{(x)}, \xi_{k j}^{(y)}\right)$ at all Gauss-Lobatto nodes. Here also, $U$ consists of three blocks of sizes $2\left(M_{1}+1\right)\left(N_{1}+1\right)$ and $2\left(N_{k}+1\right)^{2}, k=2,3$, respectively. For the same vectors $P$ and $\Lambda$ as previously, problem (5.4) is equivalent to the linear system

$$
\left(\begin{array}{lll}
A_{D} & B_{D}^{T} & 0 \\
B_{D} & 0 & E^{T} \\
0 & E & 0
\end{array}\right)\left(\begin{array}{c}
U \\
P \\
\Lambda
\end{array}\right)=\left(\begin{array}{c}
G \\
F \\
0
\end{array}\right)
$$

where the vectors $F$ and $G$ now depend on the values of $f$ and $\boldsymbol{g}$, respectively, at the Gauss-Lobatto nodes and are easier to compute than the vector $H$ of system (5.5). The size of system (5.6) is much larger than the size of system (5.5). However the matrix $A_{D}$ is now fully diagonal, with diagonal coefficients equal to the $\alpha_{k}^{-1} \rho_{k i}^{(x)} \rho_{k j}^{(y)}$, so that solving it is not necessarily more expensive. Here also, the global matrix is symmetric.

Applying an Uzawa type algorithm to eliminate the vector $U$ in (5.6) leads exactly to the same system as (5.5), so we did not use it in order to compare the numerical properties of the two systems. In all cases systems (5.5) and (5.6) are solved using the NAG routine F04JAF [17]. This routine calculates the minimal least-squares solution of $M \mathbf{x}=\mathbf{b}$ where $M$ is $m \times n$ matrix with rank $\leq n$ and $m \geq n$. The minimal leastsquares solution is the vector $\mathbf{x}$ which minimizes the norm of the residual vector $\mathbf{x}=\mathbf{b}-M \mathbf{x}$. The matrix $M$ is factorized by using singular value decomposition.

The numerical experiments deal with the following examples where, in all cases, the unique datum is the function $f, \boldsymbol{g}$ being taken equal to zero.

Example 1. The coefficients $\alpha_{k}$ satisfy

and the exact solution is given by

$$
\alpha_{1}=\alpha_{2}=\alpha_{3}=1
$$

$$
p(x, y)=\cos (\pi x) \cos (\pi y)
$$



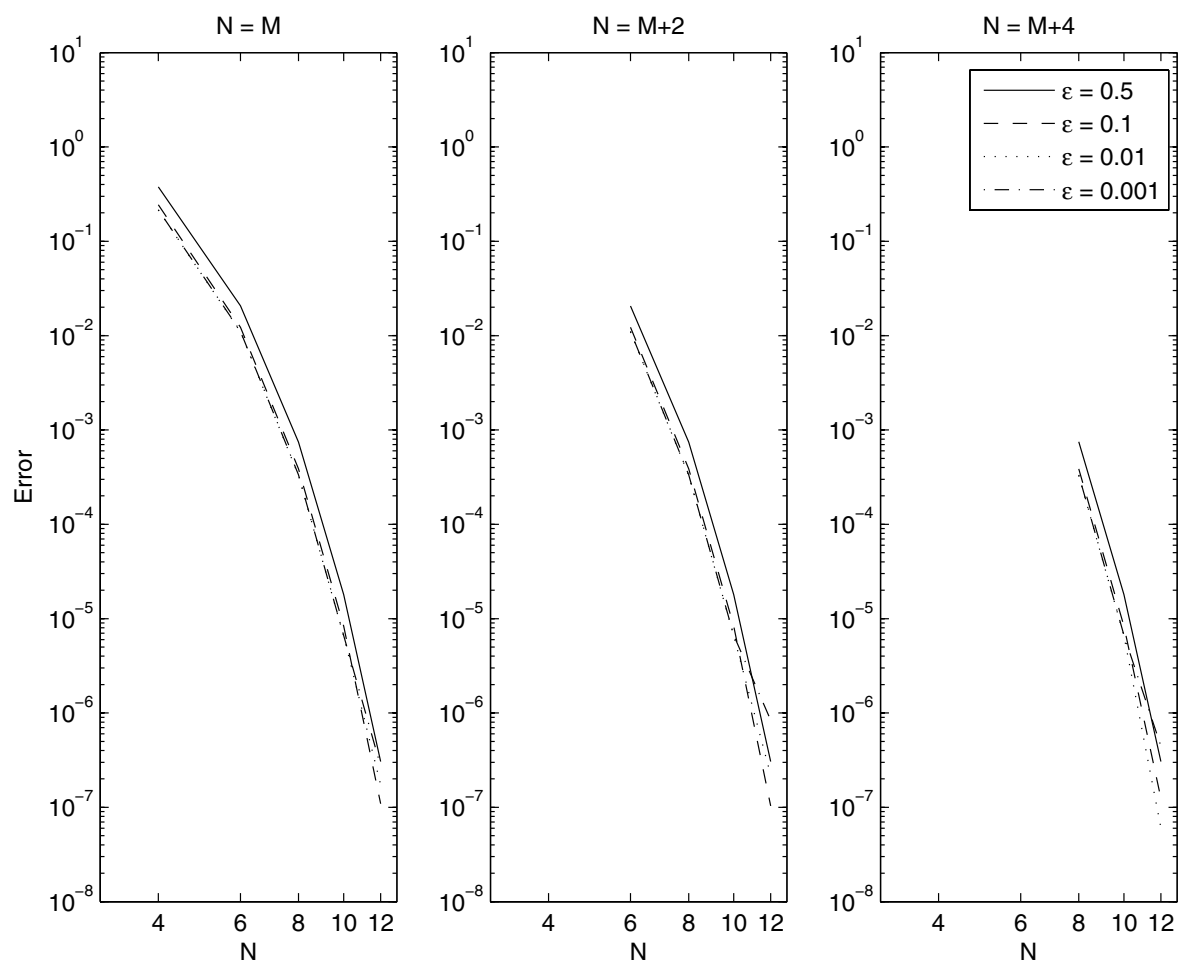

FiguRE 3. Error curves for the Laplace system in Example 1.

Example 2. The coefficients $\alpha_{k}$ again satisfy (5.7) and the exact solution is given by

$$
p(x, y)=\left(1-x^{2}\right)^{\frac{5}{2}}\left(1-y^{2}\right)^{\frac{5}{2}} .
$$

Example 3. We consider the inhomogeneous problem with

$$
\alpha_{1}=1, \quad \alpha_{2}=0.1, \quad \alpha_{3}=0.01,
$$

and datum $f$ given by

$$
f(x, y)=\cos \pi x \cos \pi y .
$$

In all cases, the error is represented as a function of a unique parameter $N$, with $N=N_{1}=N_{2}=N_{3}$, and the parameter $M=M_{1}$ is defined as a function of $N$. The error is computed in the norm defined in (2.7), it is equal to $\left\|p-p_{\delta}\right\|_{1, \alpha}$. In view of (4.24), we have chosen the mortars defined by

$$
\left.\gamma_{1}^{-}=\{0\} \times\right]-1,1-\varepsilon\left[, \quad \gamma_{2}^{-}=\right]-1,1[\times\{1-\varepsilon\},
$$

and the corresponding domains $\Omega_{m}^{-}$and $\Omega_{m}^{+}$represented in the top left part of Figure 2. However experimentation reveals that the results are independent of this choice. For simplicity, system (5.5) is called the Laplace system from now on and system (5.6) is called the Darcy system.

In Figure 3, we present the curves of the error as a function of $N$ in bilogarithmic scales for Example 1, for $\varepsilon$ taking the following values

$$
\varepsilon=0.5, \quad \varepsilon=0.1, \quad \varepsilon=0.01, \quad \varepsilon=0.001,
$$



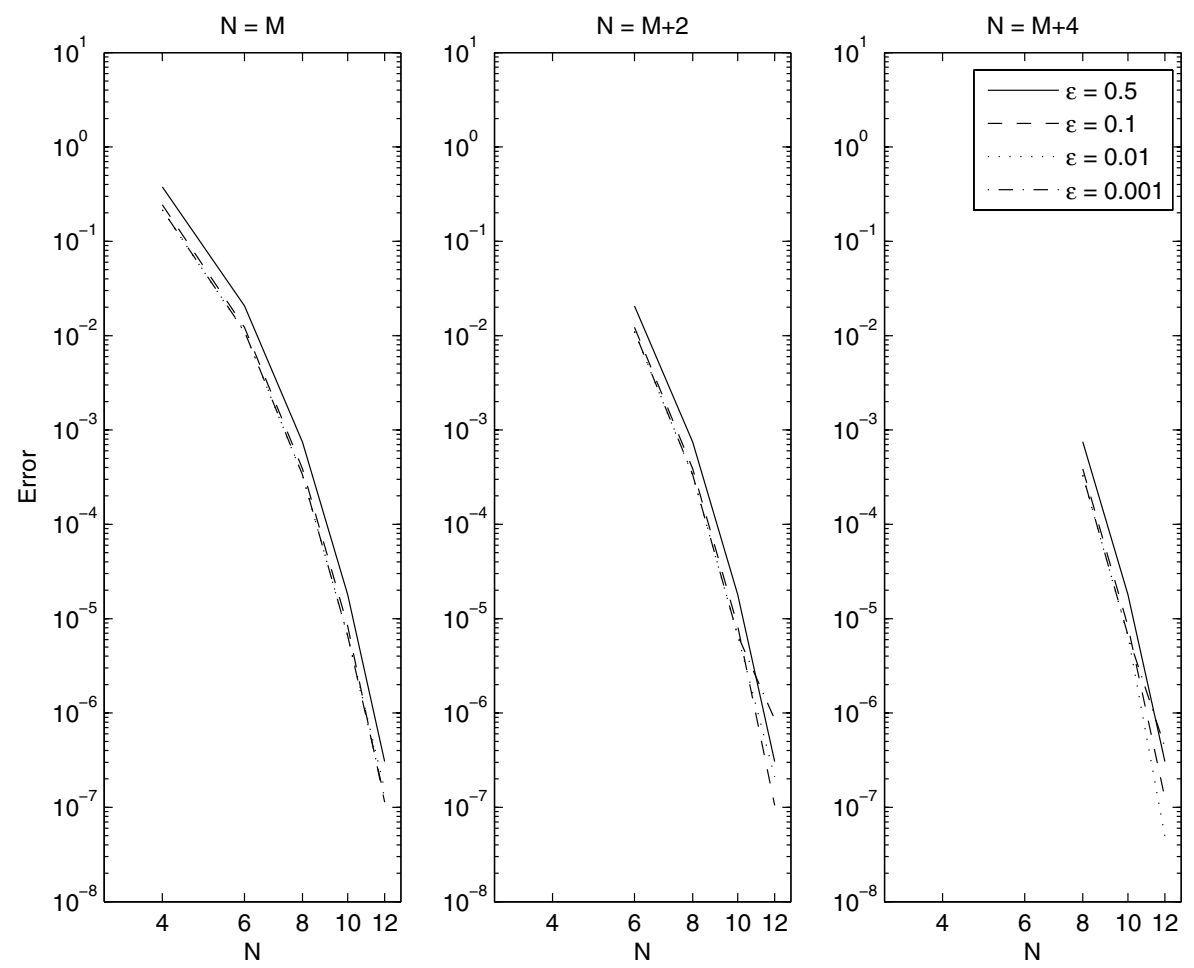

FiguRE 4. Error curves for the Darcy system in Example 1.

TABle 1. CPU times (in seconds) for Example 1.

\begin{tabular}{|c|c|c|c|c|c|}
\hline$N=M$ & 4 & 6 & 8 & 10 & 12 \\
\hline Laplace & 0.09 & 0.45 & 2.03 & 7.20 & 20.27 \\
Darcy & 1.49 & 12.92 & 62.40 & 215.96 & 608.32 \\
\hline
\end{tabular}

and in the three cases (i) $N=M$, (ii) $N=M+2$, and (iii) $N=M+4$. These results are obtained using the Laplace system. As expected, we observe exponential convergence. It can also be noted that the accuracy of the method is not significantly affected by taking fewer degrees of freedom in $\Omega_{1}$ and that it is not affected by the size of $\Omega_{1}$ which is determined by $\varepsilon$.

In Figure 4, we present the results obtained by using the Darcy system for the same Example 1. The results are identical to those obtained using the Laplace model. In order to give an indication of the computing time required, in Table 1, we present the CPU times for the solution of Example 1. These times were recorded on an IBM RS6000/43P (375 MHz). From these results it is obvious that the Laplace system is computationally cheaper than the Darcy system, at least in this simple case where all the $\alpha_{k}$ are equal. Therefore the next experiment only deals with the Laplace system.

In Figure 5, we present the curves of the error as a function of $N$ in bilogarithmic scales for Example 2, for the values of $\varepsilon$ given in (5.13) and in the three cases (i) $N=M$, (ii) $N=2 M$, and (iii) $N=3 M$ and for the Laplace system. Here, the solution is less regular than in Example 1 and, as a result, the convergence is no longer exponential. In the case $\varepsilon=0.5$, as the ratio $N / M$ increases the rate of convergence decreases. The rate of convergence does not appear to be affected as the ratio $N / M$ increases for the other values of $\varepsilon$, which seems in perfect coherence with the optimal choice of the ratio $N / M$ proposed in (4.23). 

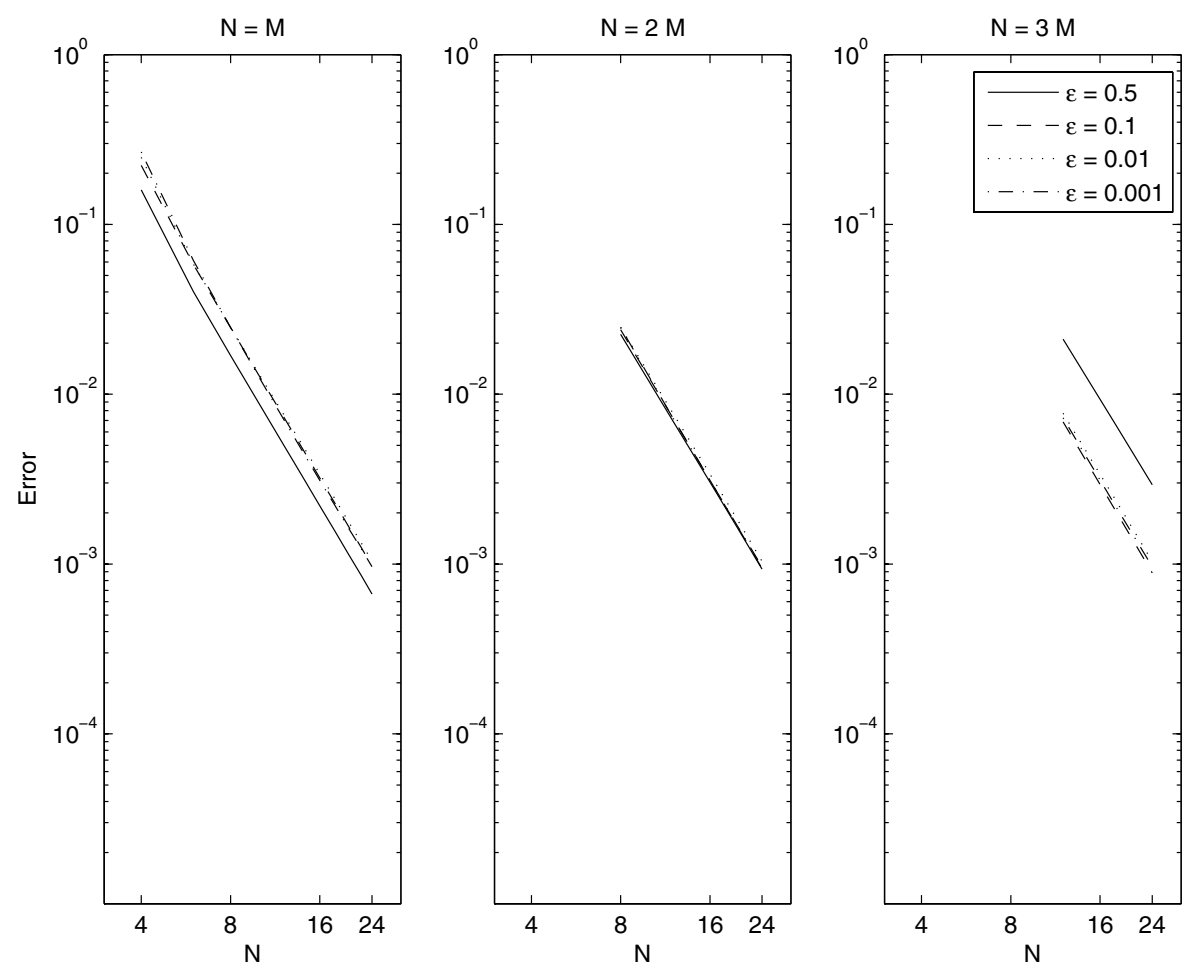

FiguRE 5. Error curves for the Laplace system in Example 2.

TABle 2. CPU times (in seconds) for Example 3.

\begin{tabular}{|c|c|c|c|c|}
\hline$M$ & 4 & 5 & 6 & 8 \\
$N$ & 8 & 10 & 12 & 16 \\
\hline Laplace & 1.23 & 4.09 & 11.15 & 59.73 \\
Darcy & 35.24 & 122.17 & 342.76 & 1995.20 \\
\hline
\end{tabular}

Finally, in Figure 6, we present the curves of the error as a function of $N$ in bilogarithmic scales for Example 3, for $\varepsilon$ now taking the following values

$$
\varepsilon=0.1, \quad \varepsilon=0.01, \quad \varepsilon=0.001,
$$

in the three cases (i) $N=M$, (ii) $N=2 M$, and (iii) $N=3 M$ and for the Laplace system. The norm of the error is now $\left\|p_{r}-p_{\delta}\right\|_{1, \alpha}$, where the reference solution $p_{r}$ is computed with $N=26$. Here, since the problem is nonhomogeneous, the convergence is no longer exponential. As $\varepsilon$ decreases so does the rate of convergence. Further, the rate of convergence for each $\varepsilon$ does not appear to be affected by the ratio $N / M$.

We do not give the results obtained for this same Example 3 and the Darcy system since they are fully similar. However, in Table 2, we present the CPU times for the solution of Example 3, there also recorded on an IBM RS6000/43P (375 MHz). The Laplace system is again cheaper than the Darcy system. So, the interest of using the Darcy system seems rather limited. 

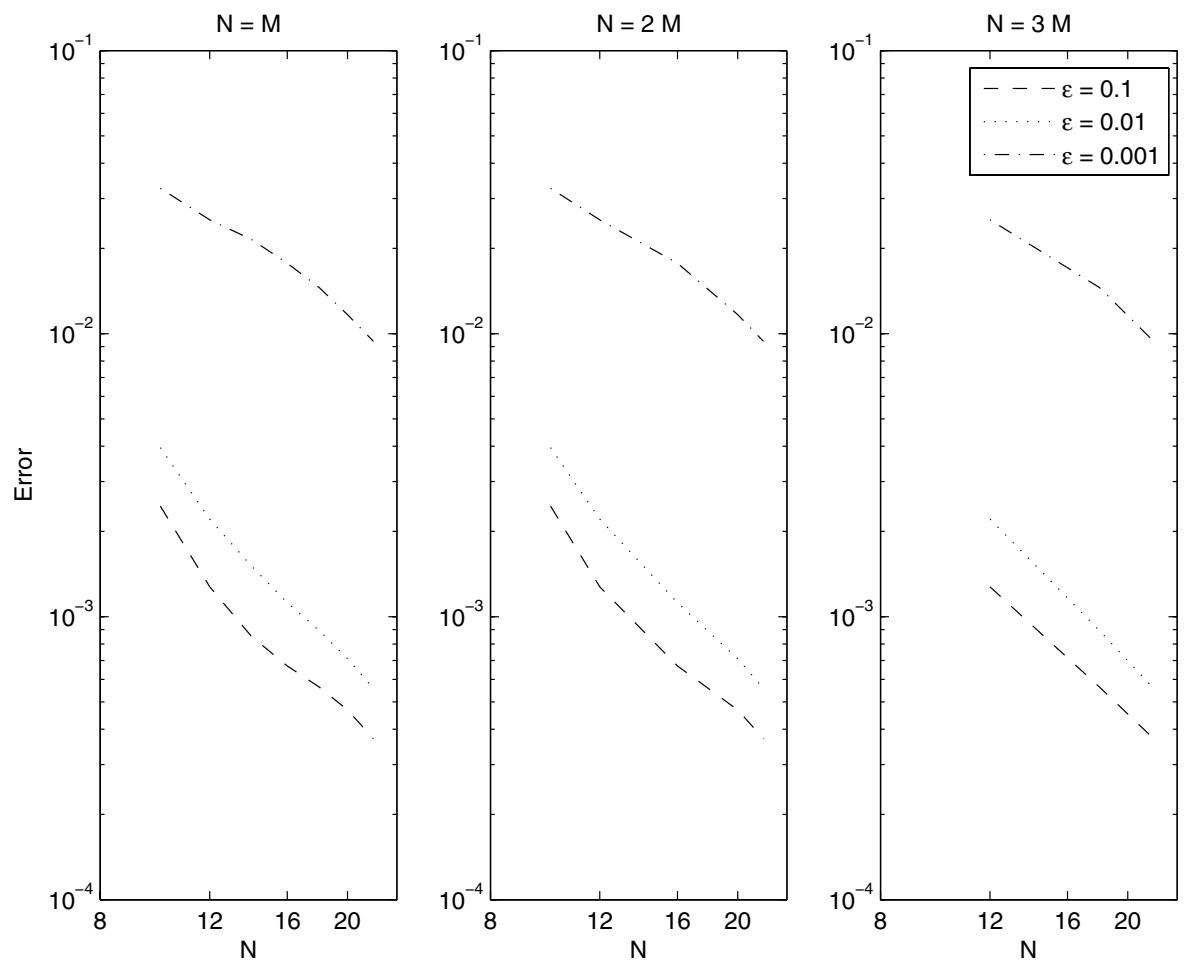

FiguRE 6. Error curves for the Laplace system in Example 3.

\section{Some CONCLUSions AND POSSIBLE EXtensions}

The results of Section 5 prove the efficiency of our approach: The accuracy of spectral methods is preserved in all computations despite the singular behaviour of the solution (see Examples 2 and 3 ) and the mortar method seems ideally suited for handling discontinuous coefficients even for anisotropic subdomains (for instance, when $\varepsilon=10^{-3}$ ). In this case, diminishing the value of $M_{1}$ for a fixed $N_{1}$ does not destroy the behaviour of the error and reduces the cost of the discretization. Note also that, in all experiments, solving the problem for the Darcy system is more expensive than solving the corresponding problem for the Laplace equation.

Several extensions of this work are possible.

(i) Extending the method to the case of dimension $d=3$ relies on the standard arguments for the threedimensional mortar method. However, it is well-known [9], Section 3, that the mortar approximation error is not fully optimal in the case of non-conforming decompositions.

(ii) The previous study can be easily applied to the case where the coefficient $\alpha$ is replaced by a tensor $A=\left(a_{r s}\right)_{1 \leq r, s \leq 2}$ which is positive definite, in the sense that

$$
\forall \boldsymbol{\xi}=\left(\xi_{1}, \xi_{2}\right) \in \mathbb{R}^{2}, \quad \sum_{r=1}^{2} \sum_{s=1}^{2} \alpha_{r s} \xi_{r} \xi_{s} \geq c \sum_{r=1}^{2} \xi_{r}^{2}
$$

Moreover, when this tensor is diagonal and the ratio $\alpha_{11} / \alpha_{22}$ is large, a much lower value of $M$ can be used without modifying the order of convergence. 
(iii) Handling complex geometries by means of curved subdomains is a little more difficult in the spectral context and requires the use of over-integration, i.e. using quadrature formulæ exact on $\mathbb{P}_{2 L-1}(-1,1)$, with $L$ larger than $N$ or $M$. We refer to [15] which includes pioneering work on this extension. The implementation of the mortar method is then more expensive (in particular, for the Darcy system, the mass matrix $A_{D}$ is no longer diagonal) but this does not increase the discretization error.

\section{REFERENCES}

[1] Y. Achdou and C. Bernardi, Un schéma de volumes ou éléments finis adaptatif pour les équations de Darcy à perméabilité variable. C.R. Acad. Sci. Paris Série I 333 (2001) 693-698.

[2] Y. Achdou, C. Bernardi and F. Coquel, A priori and a posteriori analysis of finite volume discretizations of Darcy's equations. Numer. Math. 96 (2003) 17-42.

[3] F. Ben Belgacem, The Mortar finite element method with Lagrangian multiplier. Numer. Math. 84 (1999) $173-197$.

[4] C. Bernardi and N. Chorfi, Mortar spectral element methods for elliptic equations with discontinuous coefficients. Math. Models Methods Appl. Sci. 12 (2002) 497-524.

[5] C. Bernardi and Y. Maday, Spectral Methods, in the Handbook of Numerical Analysis V, P.G. Ciarlet and J.-L. Lions Eds., North-Holland (1997) 209-485.

[6] C. Bernardi and Y. Maday, Spectral element discretizations of the Poisson equation with mixed boundary conditions. Appl. Math. Inform. 6 (2001) 1-29.

[7] C. Bernardi and R. Verfürth, Adaptive finite element methods for elliptic equations with non-smooth coefficients. Numer. Math. 85 (2000) 579-608.

[8] C. Bernardi, M. Dauge and Y. Maday, Relèvements de traces préservant les polynômes. C.R. Acad. Sci. Paris Série I 315 (1992) 333-338.

[9] C. Bernardi, Y. Maday and A.T. Patera, A new nonconforming approach to domain decomposition: the mortar element method, in Collège de France Seminar XI, H. Brezis and J.-L. Lions Eds., Pitman (1994) 13-51.

[10] C. Bernardi, Y. Maday and F. Rapetti, Discrétisations variationnelles de problèmes aux limites elliptiques, Mathématiques et Applications 45. Springer-Verlag (2004).

[11] C. Bernardi, Y. Maday and F. Rapetti, Basics and some applications of the mortar element method. GAMM - Gesellschaft für Angewandte Mathematik und Mechanik 28 (2005) 97-123.

[12] S. Bertoluzza and V. Perrier, The mortar method in the wavelet context. ESAIM: M2AN 35 (2001) 647-673.

[13] S. Clain and R. Touzani, Solution of a two-dimensional stationary induction heating problem without boundedness of the coefficients. RAIRO Modél. Math. Anal. Numér. 31 (1997) 845-870.

[14] V. Girault and P.-A. Raviart, Finite Element Methods for Navier-Stokes Equations, Theory and Algorithms. Springer-Verlag (1986).

[15] Y. Maday and E.M. Rønquist, Optimal error analysis of spectral methods with emphasis on non-constant coefficients and deformed geometries. Comput. Methods Appl. Mech. Engrg. 80 (1990) 91-115.

[16] N.G. Meyers, An $L^{p}$-estimate for the gradient of solutions of second order elliptic divergence equations. Ann. Sc. Norm. Sup. Pisa 17 (1963) 189-206.

[17] NAG Library Mark 21, The Numerical Algorithms Group Ltd, Oxford (2004). 